\title{
Nonlinear Transfer of Signal and Noise Correlations in Cortical Networks
}

\author{
Dmitry R. Lyamzin, ${ }^{1,2}$ Samuel J. Barnes, ${ }^{3}$ Roberta Donato, ${ }^{1}$ Jose A. Garcia-Lazaro, ${ }^{1}$ Tara Keck, ${ }^{3}$ and Nicholas A. Lesica ${ }^{1}$ \\ ${ }^{1}$ Ear Institute, University College London, London WC1X 8EE, United Kingdom, ${ }^{2}$ Graduate School of Systemic Neurosciences, Ludwig-Maximilians-Universität \\ Munich, 80802 Munich, Germany, and 'MRC Centre for Developmental Neurobiology, King's College London, London SE1 1UL, United Kingdom
}

\begin{abstract}
Signal and noise correlations, a prominent feature of cortical activity, reflect the structure and function of networks during sensory processing. However, in addition to reflecting network properties, correlations are also shaped by intrinsic neuronal mechanisms. Here we show that spike threshold transforms correlations by creating nonlinear interactions between signal and noise inputs; even when input noise correlation is constant, spiking noise correlation varies with both the strength and correlation of signal inputs. We characterize these effects systematically in vitro in mice and demonstrate their impact on sensory processing in vivo in gerbils. We also find that the effects of nonlinear correlation transfer on cortical responses are stronger in the synchronized state than in the desynchronized state, and show that they can be reproduced and understood in a model with a simple threshold nonlinearity. Since these effects arise from an intrinsic neuronal property, they are likely to be present across sensory systems and, thus, our results are a critical step toward a general understanding of how correlated spiking relates to the structure and function of cortical networks.
\end{abstract}

Key words: correlations; cortex; cortical state; networks; noise correlations

\section{Introduction}

Correlated neuronal activity provides a window into network structure and function over multiple timescales (Cohen and Kohn, 2011). In sensory systems, correlations can be separated into signal and noise components, where signal correlations are the correlations in the part of the neural response that is repeatable across stimulus trials, and noise correlations are the correlations in the trial-to-trial variability (Gawne and Richmond, 1993). Studies of signal and noise correlations across cortical areas typically report a positive dependency, i.e., noise correlation tends to increase with increasing signal correlation (Bair et al., 2001; Averbeck and Lee, 2003; Kohn and Smith, 2005; Ecker et al., 2014), but recent studies have shown that correlations are dynamic and can be modulated by changes in stimulus properties (Kohn and Smith, 2005; Hofer et al., 2011), adaptation (Gutnisky and Dragoi, 2008), attention (Cohen and Maunsell, 2009; Mitchell et al., 2009), brain state (Poulet and Petersen, 2008; Marguet and Harris, 2011; Ecker et al., 2014), and learning (Gu et al., 2011; Jeanne et al., 2013).

Correlations are typically attributed to shared inputs and recurrent connectivity, but they can also be shaped by intrinsic

Received Nov. 19, 2014; revised Feb. 23, 2015; accepted March 12, 2015.

Author contributions:D.R.L.,S.J.B., R.D., J.A.G.-L., T.K., and N.A.L. designed research;D.R.L.,S.J.B., R.D., J.A.G.-L., and N.A.L. performed research; D.R.L. and N.A.L. analyzed data; D.R.L., S.J.B., T.K., and N.A.L. wrote the paper.

This work was supported by the German Research Foundation, the Wellcome Trust, the Medical Research Council, the European Research Council, and the Royal Society. The authors thank M. Pachitariu, C. Stringer, K. Harris, J. Macke, L. Belliveau, and S. Hofer for helpful discussions.

The authors declare no competing financial interests.

This article is freely available online through the J Neurosci Author Open Choice option.

Correspondence should be addressed to Nicholas Lesica at the above address. E-mail: n.lesica@ucl.ac.uk.

DOI:10.1523/JNEUROSCI.4738-14.2015

Copyright $\odot 2015$ Lyamzin et al.

This is an Open Access article distributed under the terms of the Creative Commons Attribution License Creative Commons Attribution 4.0 International, which permits unrestricted use, distribution and reproduction in any medium provided that the original work is properly attributed. neuronal properties. Because of the thresholding associated with spike generation, the transfer of correlations from synaptic inputs to spiking output is highly nonlinear (Lampl et al., 1999; Binder and Powers, 2001; Dorn and Ringach, 2003; Shamir and Sompolinsky, 2004; de la Rocha et al., 2007; Shea-Brown et al., 2008; Burak et al., 2009; Tchumatchenko et al., 2010; Middleton et al., 2012). For example, even when total input correlation is constant, total output correlation can vary with changes in input strength, resulting in a positive dependency between the mean spike rate and total correlation in cortical spiking (de la Rocha et al., 2007).

The transformation of signal and noise correlations between input and output has not yet been studied directly. Many studies of sensory processing report changes in correlations that are unrelated to mean spike rate, but coincide with changes in other spiking properties (for example, trial-to-trial variability; Gutnisky and Dragoi, 2008; Cohen and Maunsell, 2009; Mitchell et al., 2009), which suggests that output correlations may depend on other properties of the input beyond just its overall strength. In fact, because correlation transfer is nonlinear, the signal and noise components in the input have the potential to interact and, as we will show, the resulting transformation of correlations goes far beyond that expected from the effects of spike threshold acting on each input separately.

\section{Materials and Methods}

In vitro recordings. Deeply anesthetized C57BL/6 mice [postnatal day $(\mathrm{P})$ $30-\mathrm{P} 40$ ] were transcardially perfused with $10 \mathrm{ml}$ of ice-cold $\left(4^{\circ} \mathrm{C}\right)$ dissection ACSF (in mm: 108 choline-Cl, $3 \mathrm{KCL}, 26 \mathrm{NaHCO}_{3} 1.25 \mathrm{NaHPO}_{4}$, $25 \mathrm{D}$-glucose, $3 \mathrm{Na}$ pyruvate, $2 \mathrm{CaCl}_{2}$, and $1 \mathrm{MgSO}_{4}$ bubbled with $95 \% \mathrm{O}_{2} /$ $5 \% \mathrm{CO}_{2}$ ). Coronal brain slices $300 \mu \mathrm{m}$ thick were cut (Vibratome 3000, Leica) from V1. Slices were incubated for $30 \mathrm{~min}$ in a holding chamber and then recordings were made at room temperature $\left(24^{\circ} \mathrm{C}\right)$ in recording ACSF (in mm: $120 \mathrm{NaCl}, 3 \mathrm{KCL}, 23 \mathrm{NaHCO}_{3}, 1.25 \mathrm{NaHPO}_{4}, 10$ 
D-glucose, $2 \mathrm{CaCl}_{2}$, and $1 \mathrm{MgSO}_{4}$ bubbled with $\left.95 \% \mathrm{O}_{2} / 5 \% \mathrm{CO}_{2}\right)$. Recordings were targeted to monocular primary visual cortex based on coordinates from the Paxinos mouse brain atlas (Paxinos and Franklin, 2004). We made whole-cell recordings from $\mathrm{L} 2 / 3$ on a custom-built microscope under differential interference contrast microscopy. L2/3 pyramidal neurons were recorded (based on spiking properties and pyramidal-shaped soma) in current-clamp mode (Multiclamp 700B, Molecular Devices). The data were acquired using Ephus freeware (VidrioTech). All recordings were low-pass filtered at $3 \mathrm{kHz}$ and digitized at $10 \mathrm{kHz}$. Patch pipettes (4-7 M $\Omega$ ) contained the following (in $\mathrm{mM}$ ): 130 $\mathrm{KMeSO}_{4}, 8 \mathrm{NaCl}, 2 \mathrm{KH} 2 \mathrm{PO}_{4}, 2$ D-glucose, and 10 HEPES. Recordings were discarded if the series resistance varied by $>15 \%$ or the resting membrane potential or input resistance varied by $>10 \%$ across the recording period.

Current stimuli. For in vitro experiments, a constant current was injected into the soma to achieve a holding potential close to the action potential threshold (typically $-55 \mathrm{mV}$ ) and zero mean signal and noise currents were injected to study the transfer of correlations. Signal and noise currents were Gaussian white noise initially created with a sampling rate of $200 \mathrm{~Hz}$ and upsampled to $10 \mathrm{kHz}$ with spline interpolation. Currents with a given set of variances and correlations were presented in blocks of 100 trials, with the signal current the same on each trial and the noise current different on each trial. Currents were delivered in $1 \mathrm{~s} \mathrm{seg-}$ ments with a $1 \mathrm{~s}$ pause between each segment. All pairs of neurons included in the analysis were recorded nonsimultaneously to ensure the only correlations were those in the injected currents. We explicitly excluded nonstationary data from the analysis. To test for nonstationarity, we calculated mean spike rates throughout a recording using a $30 \mathrm{~s} \mathrm{mov-}$ ing average; any neuron for which the minimum and maximum rates differed by $>25 \%$ during the recording was excluded from the analysis.

In vivo recordings. Adult male gerbils (70-90 g, P60-P120) were anesthetized for surgery with one of two different anesthetic combinations: (1) ketamine and xylazine for some extracellular experiments, or (2) fentanyl, medetomidine, and midazolam for some extracellular experiments and all intracellular experiments. For ketamine and xylazine, an initial injection of $1 \mathrm{ml}$ per $100 \mathrm{~g}$ body weight was given of ketamine (100 $\mathrm{mg} / \mathrm{ml})$, xylazine $(2 \% \mathrm{w} / \mathrm{v})$, and saline in a ratio of $5: 1: 19$, and the same solution was infused continuously during recording at a rate $\sim 2.5 \mu \mathrm{l} /$ min. For fentanyl, medetomidine, and midazolam, an initial injection of $0.2 \mathrm{ml}$ per $100 \mathrm{~g}$ body weight was given with fentanyl $(0.05 \mathrm{mg} / \mathrm{ml})$, medetomidine $(1 \mathrm{mg} / \mathrm{ml})$, and midazolam $(5 \mathrm{mg} / \mathrm{ml})$ in a ratio of $4: 1: 10$, and the same solution was infused continuously during recording at a rate of $\sim 0.08 \mu \mathrm{l} / \mathrm{min}$. Internal temperature was monitored and maintained at $38.7^{\circ} \mathrm{C}$ and heart rate was consistently $\sim 300-350$ beats per minute under all anesthetics. A small metal rod was mounted on the skull and used to secure the head of the animal in a stereotaxic device in a sound-attenuated chamber. A craniotomy was made over the primary auditory cortex (A1). An incision was made in the dura mater. For intracellular recordings, the cisterna magna was drained and a glass pipette filled with a $1 \mathrm{M}$ potassium acetate solution (with resistance between 70 and $100 \mathrm{M} \Omega$ ) was inserted into the brain with bridge balancing and capacitance compensation. For extracellular recordings, a multitetrode array (Neuronexus) was inserted into the brain. Intracellular recordings were made at all depths and extracellular recordings were made between 1 and $1.5 \mathrm{~mm}$ from the cortical surface (most likely in layer V; Happel et al., 2010). We explicitly excluded nonstationary data from the analysis. To test for nonstationarity, we calculated mean spike rates throughout a recording using a $30 \mathrm{~s}$ moving average; any neuron for which the minimum and maximum rates differed by $>25 \%$ during the recording was excluded from the analysis.

Spike sorting. For in vivo extracellular recordings, the procedure for the isolation of single-unit spikes consisted of the following: (1) bandpass filtering each channel between 500 and $5000 \mathrm{~Hz}$, (2) whitening each tetrode, i.e., projecting the signals from the four channels into a space in which they are uncorrelated, (3) identifying potential spikes as snippets with energy (Choi et al., 2006) that exceeded a threshold (with $\geq 0.7 \mathrm{~ms}$ between potential spikes), (4) projecting each of the snippets into the space defined by the first three principal components for each channel, (5) identifying clusters of snippets within this space using KlustaKwik (http://klustakwik.sourceforge.net) and Klusters (Hazan et al., 2006), and (6) quantifying the likelihood that each cluster represented a single unit using isolation distance (Schmitzer-Torbert et al., 2005). Isolation distance assumes that each cluster forms a multidimensional Gaussian cloud in feature space and measures, in terms of the SD of the original cluster, the increase in the size of the cluster required to double the number of snippets within it. The number of snippets in the "noise" cluster (nonisolated multiunit activity) for each tetrode was always at least as large as the number of spikes in any single-unit cluster. Only single-unit clusters with an isolation distance of $>20$ were analyzed.

Auditory stimuli. For in vivo experiments, sounds were generated with a $48 \mathrm{kHz}$ sampling rate, attenuated, and delivered to speakers. Speakers (Etymotic ER2) coupled to tubes were inserted into both ear canals for diotic sound presentation along with microphones for calibration. The frequency response of these speakers measured at the entrance of the ear canal was flat ( $\pm 5 \mathrm{~dB}$ SPL) between 0.2 and $5 \mathrm{kHz}$.

In all intracellular experiments and some extracellular experiments, frequency-modulated (FM) sounds were presented that consisted of a signal component that was the same on every trial and a noise component that was different on every trial. The signal and noise components were created by drawing numbers between 7 and 13 at random from a uniform distribution and scaled to achieve the desired signal-to-noise ratio (SNR), measured as the ratio of their variances, while keeping the variance of their sum constant. The sum of the signal and noise components was then used as powers of two to create a set of frequencies (falling mostly between 128 and $8192 \mathrm{~Hz}$ ). The resulting set of frequencies was used to specify the frequency of a constant amplitude tone at $10 \mathrm{~ms}$ intervals so that each $10 \mathrm{~ms}$ segment of the sound was a frequency modulation that started at the final frequency of the previous segment and moved linearly to a new frequency over the $10 \mathrm{~ms}$ (see Fig. $2 \mathrm{~A}$, spectrogram). For intracellular experiments, three different signal components were used, yielding six different possible pairings of signal components. The correlation between the different signal components was 1 for the three pairings of identical signals, and $0.95,0.8$, and 0.5 for the three possible pairings of different signals. For extracellular experiments, four different signal components were used, yielding 10 different possible pairings of signal components. The correlation between the different signal components was 1 for the four pairings of identical signals, and $0.95,0.9,0.8,0.65,0.5$, and 0.3 for the six possible pairings of different signals. For all experiments, two different noise components were used, with a correlation of 0.95 between them. All combinations of signal and noise components were presented with an SNR of 3, yielding a set of sounds with fixed SNR and noise correlation but varying signal correlation, and one of the signal components was presented with each noise component at SNRs of 0.1 (extracellular only), 0.3 (extracellular only), 1, 3,10 , and 30 , yielding a set of sounds with fixed signal and noise correlations, but varying SNRs. All sounds were presented over 100 trials of $2.5 \mathrm{~s}$ each with an intensity of either 55 or $65 \mathrm{~dB}$ SPL. In some extracellular experiments, 256 repeated trials of a $2.5 \mathrm{~s}$ segment of female speech from the University College London SCRIBE (Spoken Corpus of British English) database (http://www.phon.ucl.ac.uk/resource/scribe) was presented at a peak intensity of $75 \mathrm{~dB}$ SPL.

Analysis of membrane potential. The analyses of membrane potentials recorded in vitro and in vivo were performed as follows: the membrane potential for neuron $p$ on trial $i$ in each $50 \mathrm{~ms}$ time bin $t, v_{i}^{p}(t)$, was obtained by first removing action potentials and then downsampling from $10 \mathrm{kHz}$ by computing the mean value in each time bin. The total membrane potential variance for each neuron $p$ was computed as the average variance of these signals across trials, $\left\langle\operatorname{var}\left(v_{i}^{p}\right)\right\rangle_{i}$, and the total membrane potential correlation between two neurons $p$ and $q$ was calculated as the correlation coefficient between membrane potentials on the same trials:

$$
\rho_{v}=\frac{\left\langle\operatorname{cov}\left(v_{i}^{p}, v_{i}^{q}\right)\right\rangle_{i}}{\sqrt{\left\langle\operatorname{var}\left(v_{i}^{p}\right)\right\rangle_{i}\left\langle\operatorname{var}\left(v_{i}^{q}\right)\right\rangle_{i}}}
$$

The signal component of the membrane potential was estimated from the mean membrane potential averaged across all trials. The membrane potential signal variance for each neuron $p$ was computed as the variance of the signal component $\operatorname{var}\left(\left\langle v_{i}^{p}\right\rangle_{i}\right)$ and the membrane potential signal 
correlation between two neurons $p$ and $q$ was calculated as the correlation coefficient between signal components:

$$
\rho_{s}=\frac{\operatorname{cov}\left(\left\langle v_{i}^{p}\right\rangle_{i},\left\langle v_{i}^{q}\right\rangle_{i}\right)}{\sqrt{\operatorname{var}\left(\left\langle v_{i}^{p}\right\rangle_{i}\right) \operatorname{var}\left(\left\langle v_{i}^{q}\right\rangle_{i}\right)}}
$$

The noise component of the membrane potential was estimated from the deviation of the membrane potential on each trial from the mean averaged across all trials. The membrane potential noise variance for each neuron $p$ was computed as the average variance of these noise components across trials $\left\langle\operatorname{var}\left(v_{i}^{p}-\left\langle v_{i}^{p}\right\rangle_{i}\right)\right\rangle_{i}$ and membrane potential noise correlation between two neurons $p$ and $q$ was calculated as the correlation coefficient between these noise components:

$$
\rho_{n}=\frac{\left\langle\operatorname{cov}\left(v_{i}^{p}-\left\langle v_{i}^{p}\right\rangle_{i}, v_{i}^{q}-\left\langle v_{i}^{q}\right\rangle_{i}\right)\right\rangle_{i}}{\sqrt{\left\langle\operatorname{var}\left(v_{i}^{p}-\left\langle v_{i}^{p}\right\rangle_{i}\right)\right\rangle_{i}\left\langle\operatorname{var}\left(v_{i}^{q}-\left\langle v_{i}^{q}\right\rangle_{i}\right)\right\rangle_{i}}}
$$

Analysis of spiking. The analyses of spiking recorded in vitro and in vivo or simulated from the leaky integrate-and-fire (LIF) model (see below) was performed as follows: spiking was considered as spike count vectors with $50 \mathrm{~ms}$ time bins (for experimental data) or with time bins specified by the response timescale $\tau_{\mathrm{r}}$ (for simulated data) with $r_{i}^{p}(t)=N$ representing the number of action potentials fired by neuron $p$ in time bin $t$ on trial $i$. Our measure of spiking trial-to-trial variability is the SNR, which was computed as follows:

$$
\mathrm{SNR}=\frac{\operatorname{var}(\bar{r})}{\left\langle\operatorname{var}\left(r_{i}-\bar{r}\right)\right\rangle_{i}}
$$

where $\bar{r}$ is the average spiking across trials [the peristimulus time histo$\operatorname{gram}(\mathrm{PSTH})]$, and $\operatorname{var}\left(r_{i}-\overline{\mathrm{r}}\right)$ is the variance of the deviations from the PSTH on trial $i$.

The total spiking correlation between the spiking of two neurons $p$ and $q$ was calculated as the correlation coefficient between spiking on the same trials:

$$
\rho_{r}=\frac{\left\langle\operatorname{cov}\left(r_{i}^{p}, r_{i}^{q}\right)\right\rangle_{i}}{\sqrt{\left\langle\operatorname{var}\left(r_{i}^{p}\right)\right\rangle_{i}\left\langle\operatorname{var}\left(r_{i}^{q}\right)\right\rangle_{i}}}
$$

The spike train signal correlation between spiking of two neurons $p$ and $q$ was calculated as the correlation between spiking on different trials:

$$
\rho_{r}^{s i g}=\frac{\left\langle\operatorname{cov}\left(r_{i}^{p}, r_{j}^{q}\right)\right\rangle_{i \neq j}}{\sqrt{\left\langle\operatorname{var}\left(r_{i}^{p}\right)\right\rangle_{i}\left\langle\operatorname{var}\left(r_{i}^{q}\right)\right\rangle_{i}}}
$$

The spike train noise correlation was calculated as the difference between total and signal correlations:

$$
\rho_{r}^{\text {noise }}=\rho_{r}-\rho_{r}^{\text {sig }}=\frac{\left\langle\operatorname{cov}\left(r_{i}^{p}, r_{i}^{q}\right)\right\rangle_{i}-\left\langle\operatorname{cov}\left(r_{i}^{p}, r_{j}^{q}\right)\right\rangle_{i \neq j}}{\sqrt{\left\langle\operatorname{var}\left(r_{i}^{p}\right)\right\rangle_{i}\left\langle\operatorname{var}\left(r_{i}^{q}\right)\right\rangle_{i}}}
$$

Partial correlations between spiking properties were computed using the "partialcorr" function in Matlab, which computes the partial correlation between $X$ and $Y$ controlling for $Z$ as the correlation between the residuals resulting from the regression of $X$ on $Z$ and the residuals resulting from the regression of $Y$ on $Z$ (using a recursive algorithm for multidimensional $Z$ ).

A dichotomized Gaussian model for studying correlation transfer. We have previously developed a dichotomized Gaussian framework to generate spiking with specified signal and noise correlations (Lyamzin et al., 2010, 2012). For a pair of neurons with identical input signal and noise variances, binary output spiking is obtained by thresholding the sum of signal and noise inputs in each time bin $t$ :

$$
r(t)=\left\{\begin{array}{ccc}
1 & \text { if } & s(t)+n(t)>1 \\
0 & \text { else }
\end{array}\right.
$$

with signal input $s \sim \mathcal{N}\left(0, \sigma_{s}^{2}\right)$ assumed to be the same on each trial, noise input $n \sim \mathcal{N}\left(0, \sigma_{n}^{2}\right)$ assumed to be different on each trial, and correlations $\rho_{\mathrm{s}}$ and $\rho_{\mathrm{n}}$ between the signal and noise inputs to the two neurons.

Correlations in dichotomized Gaussian model spiking for single time bins. The total, signal and noise correlations in the output of the dichotomized
Gaussian (DG) model can be computed directly from the input variances and correlations. For two neurons $a$ and $b$ with identical input signal and noise variances, the output correlation can be written as follows:

$$
\begin{aligned}
\rho_{r}=\frac{\operatorname{cov}\left(r_{a}, r_{b}\right)}{\sqrt{\operatorname{var}\left(r_{a}\right) \operatorname{var}\left(r_{b}\right)}}=\frac{\operatorname{cov}\left(r_{a}, r_{b}\right)}{\operatorname{var}\left(r_{a}\right)}=\frac{\mathrm{E}\left[r_{a} r_{b}\right]-\mathrm{E}\left[r_{a}\right] \mathrm{E}\left[r_{b}\right]}{\mathrm{E}\left[r_{a}^{2}\right]-\mathrm{E}\left[r_{a}\right]^{2}} \\
=\frac{\mathrm{E}\left[r_{a} r_{b}\right]-\mathrm{E}\left[r_{a}\right]^{2}}{\mathrm{E}\left[r_{a}^{2}\right]-\mathrm{E}\left[r_{a}\right]^{2}}
\end{aligned}
$$

For responses in a single time bin, both $\mathrm{E}\left[r_{a}\right]$ and $\mathrm{E}\left[r_{a}^{2}\right]=\Phi\left(-1, \sigma_{s}^{2}+\sigma_{n}^{2}\right)$, where $\Phi$ is the value of the cumulative distribution function (CDF) for a Gaussian with zero mean and variance $\sigma_{s}^{2}+\sigma_{n}^{2}$ evaluated at -1 .

For responses on the same trial, $\mathrm{E}\left[r_{a} r_{b}\right]=\Phi_{2}\left(-1, \Sigma_{\text {tot }}\right)$, where $\Phi_{2}$ is the value of the CDF for a bivariate Gaussian with zero mean and covariance:

$$
\Sigma_{t o t}=\left[\begin{array}{cc}
\sigma_{s}^{2}+\sigma_{n}^{2} & \sigma_{s}^{2} \rho_{s}+\sigma_{n}^{2} \rho_{n} \\
\sigma_{s}^{2} \rho_{s}+\sigma_{n}^{2} \rho_{n} & \sigma_{s}^{2}+\sigma_{n}^{2}
\end{array}\right]
$$

evaluated at -1 .

For responses on different trials, $\mathrm{E}\left[r_{a} r_{b}\right]=\Phi_{2}\left(-1, \Sigma_{s i g}\right)$, where

$$
\Sigma_{s i g}=\left[\begin{array}{cc}
\sigma_{s}^{2}+\sigma_{n}^{2} & \sigma_{s}^{2} \rho_{s} \\
\sigma_{s}^{2} \rho_{s} & \sigma_{s}^{2}+\sigma_{n}^{2}
\end{array}\right]
$$

Thus, the output total correlation, which is the correlation between responses on the same trial, is given by the following:

$$
\rho_{r}^{\text {tot }}=\frac{\Phi_{2}\left(-1, \Sigma_{t o t}\right)-\Phi\left(-1, \sigma_{s}^{2}+\sigma_{n}^{2}\right)^{2}}{\Phi\left(-1, \sigma_{s}^{2}+\sigma_{n}^{2}\right)-\Phi\left(-1, \sigma_{s}^{2}+\sigma_{n}^{2}\right)^{2}}
$$

The output signal correlation, which we define as the correlation between responses on different trials, is given by the following:

$$
\rho_{r}^{s i g}=\frac{\Phi_{2}\left(-1, \Sigma_{s i g}\right)-\Phi\left(-1, \sigma_{s}^{2}+\sigma_{n}^{2}\right)^{2}}{\Phi\left(-1, \sigma_{s}^{2}+\sigma_{n}^{2}\right)-\Phi\left(-1, \sigma_{s}^{2}+\sigma_{n}^{2}\right)^{2}}
$$

Meanwhile, the output noise correlation, which we define as the difference between the total correlation and the signal correlation, is given by the following:

$$
\rho_{r}^{\text {noise }}=\rho_{r}^{\text {tot }}-\rho_{r}^{s i g}=\frac{\Phi_{2}\left(-1, \Sigma_{t o t}\right)-\Phi_{2}\left(-1, \Sigma_{s i g}\right)}{\Phi\left(-1, \sigma_{s}^{2}+\sigma_{n}^{2}\right)-\Phi\left(-1, \sigma_{s}^{2}+\sigma_{n}^{2}\right)^{2}}
$$

Note these are the same as Equations 6-8 in the Results.

Mutual information in DG model spiking for single time bins. The mutual information between the output of the DG model and the input signal $s$ can be computed directly from the input variances and correlations. Mutual information is defined as follows:

$I(r ; s)=H(r)-H(r \mid s)=-\sum_{r} p(r) \log _{2} p(r)$

$$
+\sum_{s} p(s) \sum_{r} p(r \mid s) \log _{2} p(r \mid s)
$$

For two neurons $a$ and $b$ with identical input variances, the conditional probability of a joint binary response in a single time bin is $p(r \mid s)=\Phi_{2}\left(\theta, \Sigma_{n}\right)$, where $\Phi_{2}$ is the value of the CDF for a bivariate Gaussian with zero mean and covariance $\Sigma_{n}=\left[\begin{array}{cc}\sigma_{n}^{2} & \sigma_{n}^{2} \rho_{n} \\ \sigma_{n}^{2} \rho_{n} & \sigma_{n}^{2}\end{array}\right]$ evaluated at $\theta=\left[\begin{array}{c}\theta_{a} \\ \theta_{b}\end{array}\right]$, where $\theta_{i \in\{a, b\}}=\left\{\begin{array}{ccc}1-s_{i} & \text { if } & r_{i}=0 \\ -1+s_{i} & \text { if } & r_{i}=1 \\ -s^{2} & & \end{array}\right.$ and the stimulus probability is $p(s)=\frac{1}{\sigma_{s} \sqrt{2 \pi}} e^{\frac{-s^{2}}{2 \sigma_{s}^{2}}}$. We computed the information by summing these probabilities over all values of $r$ and $s$.

Mutual information breakdown in DG model spiking for single time bins. We also measured the impact of signal and noise correlations on information using an information breakdown approach (Pola et al., 2003). We computed the information loss due to signal correlations as follows: 


$$
\begin{aligned}
I_{\text {sig-sim }}(r ; s)=H_{\text {ind }}(r)-H_{\text {lin }}(r)=-\sum_{r} p_{\text {ind }}(r) & \log _{2} p_{\text {ind }}(r) \\
& +\sum_{r} p_{\text {lin }}(r) \log _{2} p_{\text {lin }}(r)
\end{aligned}
$$

where $p_{\text {ind }}(r)$ is the probability of a joint binary response, assuming there are no noise correlations between cells, and $p_{\text {lin }}(r)$ is the probability of a joint binary response, assuming there are no signal or noise correlations between cells. We computed the contribution of stimulus-independent noise correlations to information as follows:

$$
\begin{aligned}
I_{\text {cor-ind }}(r ; s)=H_{\text {ind }}(r)-\chi(r)=-\sum_{r} p_{\text {ind }}(r) \log _{2} p_{\text {ind }}(r) & \\
& +\sum_{r} p(r) \log _{2} p_{\text {ind }}(r)
\end{aligned}
$$

and the contribution of stimulus-dependent noise correlations to information as follows:

$$
\begin{array}{r}
I_{\text {cor-dep }}(r ; s)=H(r)-H(r \mid s)-\chi(r)+H_{\text {ind }}(r \mid s) \\
\begin{array}{r}
=-\sum_{r} p(r) \log _{2} p(r)+\sum_{s} p(s) \sum_{r} p(r \mid s) \log _{2} p(r \mid s)+\sum_{r} p(r) \log _{2} p_{\text {ind }}(r) \\
-\sum_{s} p(s) \sum_{r} p_{\text {ind }}(r \mid s) \log _{2} p_{\text {ind }}(r \mid s)
\end{array}
\end{array}
$$

We calculated each of these information breakdown quantities from simulated DG model spiking using the Direct Method via infoToolbox for Matlab (Magri et al., 2009) with bias correction via the shuffling method and quadratic extrapolation (Panzeri et al., 2007). We verified that we simulated responses to a sufficient number of trials and stimuli to achieve stable results.

Generating DG model spiking with specified output correlations. To examine the effects of nonlinear correlation transfer on information, we artificially decoupled changes in output correlations from changes in input properties using the DG model. For example, under normal conditions, an increase in input signal correlation increases both output signal and noise correlation. To remove the effects of the crossover between signal and noise correlations, we generated spiking with the same increase in output signal correlation, but no change in output noise correlation. This was achieved by decreasing the input noise correlation by the amount required to offset the increase in output noise correlation caused by the increase in input signal correlation. An analogous approach was used to keep output correlations fixed while changing input SNR.

An LIF model for studying correlation transfer. To study the nonlinear transfer of signal and noise correlations across a range of timescales, we used an LIF model, where the membrane potential $v(t)$ for each cell at each time step is given by the following:

$$
\tau_{m} \frac{d v(t)}{d t}=-v(t)+s(t)+n(t)
$$

with signal input $s \sim \mathcal{N}\left(0, \sigma_{s}^{2}\right)$ assumed to be the same on each trial, and noise input $n \sim \mathcal{N}\left(0, \sigma_{n}^{2}\right)$ assumed to be different on each trial as in the DG model. We added temporal correlations to the signal and noise inputs so that input signal $s$ had autocorrelation $e^{-t / \tau_{s}}$ and cross-correlation $\rho_{s} e^{-t / \tau_{s}}$, and the input noise $n$ had autocorrelation $e^{-t / \tau_{n}}$ and crosscorrelation $\rho_{n} e^{-t / \tau_{n}}$. We generated binary spike trains as in the DG model:

$$
r(t)=\left\{\begin{array}{ccc}
1 & \text { if } & v(t)>1 \\
0 & \text { else }
\end{array}\right.
$$

We used a membrane time constant $\tau_{\mathrm{m}}=10 \mathrm{~ms}$, imposed a refractory period during which $v(t)$ was fixed at 0 for $2 \mathrm{~ms}$ after each spike, and simulated model responses with $0.1 \mathrm{~ms}$ time steps.

\section{Results}

Nonlinear transfer of signal and noise correlations in vitro Figure $1 A$ illustrates two fundamental properties of correlation transfer that arise from thresholding. These have been described previously (Lampl et al., 1999; Binder and Powers, 2001; Dorn and Ringach, 2003; Shamir and Sompolinsky, 2004; de la Rocha et al., 2007; Shea-Brown et al., 2008; Burak et al., 2009; Tchumatchenko et al., 2010; Middleton et al., 2012) and underlie the effects of spike threshold on signal and noise correlations, which we will explore below. The first of these properties, which we call variance gain, is that the correlation between the spiking of a pair of neurons increases as the strength (which we measure as variance) of their inputs is increased, even when the correlation in their inputs is constant. This increase in output correlation with increasing input variance is sublinear, i.e., each incremental increase in input variance results in a successively smaller increase in output correlation. The second fundamental property of correlation transfer, which we call correlation gain, is that output correlation increases supralinearly with increasing input correlation when all other input properties are held constant. In this study, we investigate the impact of variance gain and correlation gain on the transfer of signal and noise correlations in cortical networks during sensory processing.

Because the transfer of correlations from input to output is nonlinear, the signal and noise components of inputs during sensory processing have the potential to interact with results that cannot be predicted from the effects of variance gain and correlation gain acting on each component separately. To characterize these interactions, we injected Gaussian white noise currents with specified variances and correlations into layer $2 / 3$ pyramidal neurons in mouse visual cortex in vitro. To simulate a typical study of sensory processing with repeated presentations of an identical stimulus, we injected currents with a signal component that was the same on each trial and a noise component that was different on each trial (Fig. 1B). To eliminate any correlations due to connections between neurons and ensure that the only signal and noise correlations in the inputs to the neurons were those that we controlled, we recorded neurons one at a time and compared input and output correlations between "virtual pairs," i.e., pairs recorded nonsimultaneously with noise correlations created by the injected currents. To account for differences in membrane filtering properties between neurons and isolate the effects of the thresholding associated with spike generation on correlation transfer, we measured input variances and correlations from the membrane potential fluctuations (with spikes removed) measured at the soma, rather than the injected currents themselves (Fig. 1C). We report only results based on measurements of membrane potential and spiking correlations with $50 \mathrm{~ms}$ time bins, but all of the effects that we observed were evident across a wide range of timescales (see Fig. 5).

To isolate the effects of different input properties, we systematically changed either the variance of the signal or noise component or the correlation between the signal or noise components for each pair while keeping other input properties constant. First, to examine the effects of variance gain on signal and noise correlations, we injected currents with fixed signal and noise correlations and changed the input variances. Figure $1 D$ shows the output signal and noise correlations as a function of the input signal variance. As input signal variance increases, output signal correlation also increases, as might be expected from variance gain. More surprisingly, output noise correlation decreases with increasing input signal variance, even though input noise variance and correlation are constant.

The effects of input noise variance on output correlations mirror those of input signal variance: an increase in the input noise variance causes an increase in the output noise correlation and a decrease in the output signal correlation (data not shown). To separate effects related to input signal and noise variance from those related to total input variance (and, thus, mean spike rate), we also varied the rela- 
A

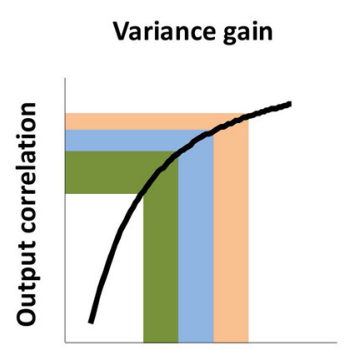

Input variance
B

Whole-cell recordings in vitro

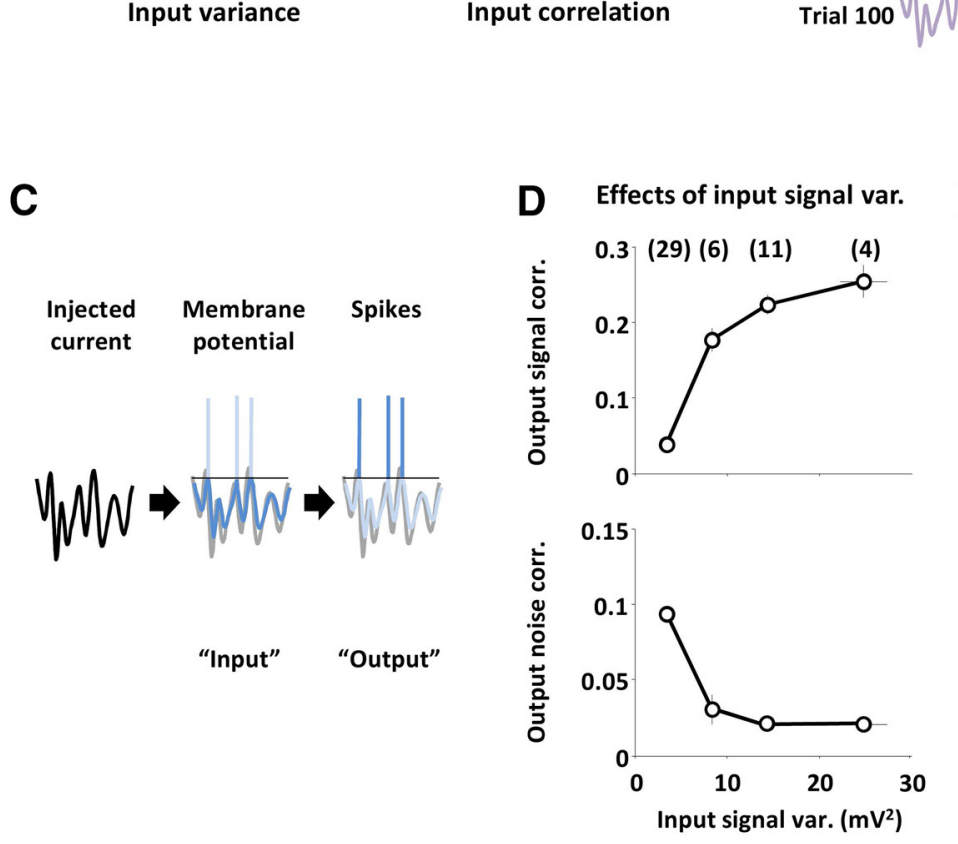

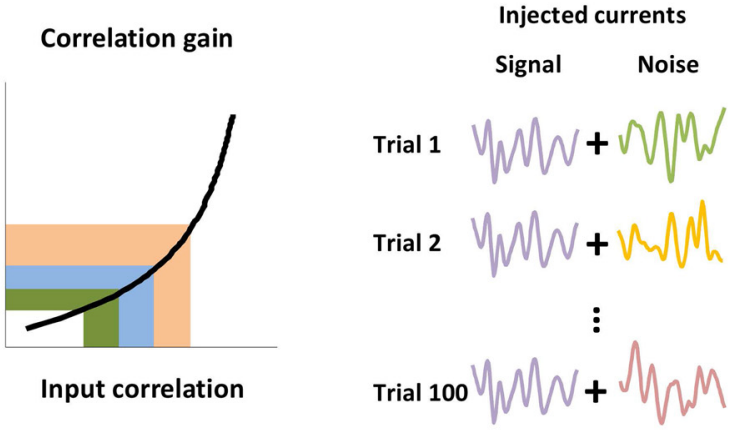
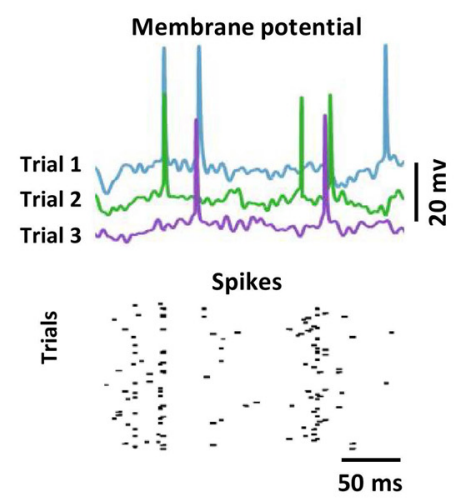

C

“Input" “Output"
D Effects of input signal var.

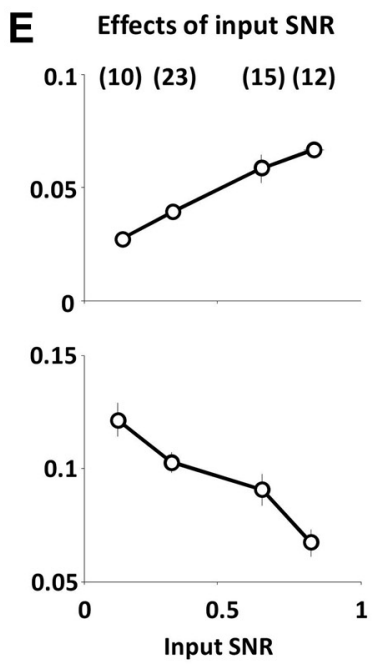

F Effects of input signal corr.
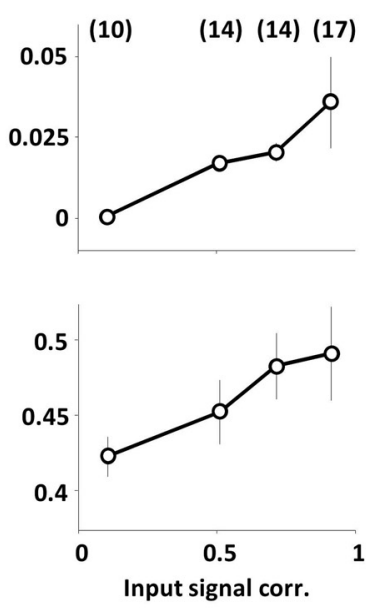

Figure 1. Nonlinear transfer of signal and noise correlations in vitro. $\boldsymbol{A}$, Schematic diagrams illustrating variance gain and correlation gain. Each colored area corresponds to an equal increment on the horizontal axis. $\boldsymbol{B}$, To mimic a typical sensory processing experiment with repeated presentations of an identical stimulus, we injected currents with a signal component that was the same on each trial, and a noise component that was different on each trial. Example membrane potential traces and spiking across trials for a typical neuron are shown. Action potentials were clipped for plotting. C, We injected Gaussian white noise currents into layer 2/3 pyramidal neurons in mouse V1 in vitro and analyzed the dependence of output signal and noise correlations on the variance and correlation of signal and noise inputs. We defined the input as the membrane potential fluctuations at the soma and the output as spike counts in 50 ms time bins. $D$, The input signal variances and output signal and noise correlations (top and bottom, respectively) that resulted from injecting currents with changing signal variance and fixed noise variance, signal correlation, and noise correlation. Pairs were grouped by input signal variance. The input signal variance for a pair of neurons was computed as the geometric mean of the input signal variances for each neuron. The numbers in parentheses above each data point indicate the number of pairs in each group. Error bars indicate $95 \%$ confidence intervals on the median value across all pairs in a particular group (estimated via bootstrapping). The mean input noise variance, input signal correlation, and input noise correlation were $20 \mathrm{mV}^{2}, 0.4$, and 0.25 . $\boldsymbol{E}$, The input SNRs and output signal and noise correlations that resulted from injecting currents with changing SNR and fixed correlations. Pairs were grouped by input SNR. The input SNR for a pair of neurons was computed as the geometric mean of the SNRs for each neuron. The mean values of the total input variance, input signal correlation, and input noise correlation were $25 \mathrm{mV}{ }^{2}, 0.5$, and 0.35 . $F$, The input signal correlations and output signal and noise correlations that resulted from injecting currents with changing signal correlation and fixed signal variance, noise variance, and noise correlation. Pairs were grouped by input signal correlation. The mean input signal variance, input noise variance, and input noise correlation were $4 \mathrm{mV}^{2}, 8 \mathrm{mV}^{2}$, and 0.8 .

tive strength of the injected signal and noise currents (and, thus, the trial-to-trial variability of the input and output) while keeping the total input variance constant. As shown in Figure $1 E$, changes in the input SNR have opposing effects on output signal and noise correlations: as input SNR is increased, output signal correlation is increased and output noise correlation is decreased. These results demonstrate that interactions between signal and noise inputs due to intrinsic nonlinearities can alter spiking correlations, even when input correlations are constant.

Next, to examine the effects of correlation gain on signal and noise correlations, we injected currents with fixed signal and noise variances and varied the signal correlation. As shown in Figure $1 F$, an increase in input signal correlation causes an increase in both output signal and noise correlations, even when input noise correlation is held constant. This dependency of output noise correlation on input signal correlation arises from the supralinearity of correlation gain. This crossover between signal and noise correlations does not occur when only the input noise correlation is changed (input noise correlation affects output noise correlation, but cannot affect output signal correlation because noise correlations are eliminated through averaging or shuffling across trials before computing signal correlations).

Nonlinear transfer of signal and noise correlations in vivo Our in vitro results suggest that the thresholding associated with spike generation can significantly transform the signal and noise correlations between a pair of neurons, with changes in the strength of signal or noise inputs creating opposing changes in output signal and noise correlations due to variance gain, and 
crossover between input signal correlation and output noise correlation due to correlation gain. To examine whether these effects were also evident during sensory processing in vivo, we examined responses to FM sounds using intracellular recordings in the primary auditory cortex (A1) in anesthetized gerbils. Unlike current injection, manipulation of a sensory stimulus in vivo does not allow for complete control over the input signal and noise variances and correlations for a given pair of neurons. To minimize the impact of the inputs that we could not control, we used the same virtual pair approach that we used in vitro (i.e., we compared only responses that were recorded nonsimultaneously), imposing both signal and noise correlations through the FM sounds. As with our injected currents, the FM sounds had a signal component that was the same on each trial and a noise component that was different on each trial, and we changed the variances and correlations of these components systematically. The spectrogram of one of the FM sounds is shown in Figure $2 A$ (see Materials and Methods for additional details about how we constructed the sounds). To eliminate any confounds due to differences in mean spike rate, frequency preference, etc., between neurons, we formed pairs using only responses from the same neuron.

Based on the effects that we observed in vitro, we expected that (1) an increase in the SNR of the sounds would increase the input SNR to both neurons in a pair, causing an increase in their output signal correlation and a decrease in their output noise correlation (Fig. 1E), and (2) an increase in the correlation between the signal components of the sounds would increase the input signal correlation between neurons in a pair, causing an increase in both their output signal and noise correlations (Fig. $1 F$ ).

We first examined the relationships between input SNR and output signal and noise correlations that resulted from changing the SNR of the sounds while keeping the correlations in the sounds fixed. Figure $2 B$ shows the membrane potential and spiking responses of a typical neuron to the same sounds with two different SNRs; as expected, the trial-to-trial variability of the responses increased as the SNR of the sounds was decreased. The output (spiking) signal and noise correlations as a function of the input (membrane potential) SNR for the pair formed from the responses of this neuron are shown in Figure $2 C$; with increasing input SNR, output signal correlation increased and output noise correlation decreased, which is consistent with the results of our in vitro experiments. We observed similar effects in all of our intracellular recordings $(n=6)$ : as shown in Figure $2 D$, the best linear fit between input SNR and output signal correlation had a positive slope for all pairs, while the best linear fit between input SNR and output noise correlation had a negative slope for all pairs. We also confirmed that the same effects were evident in a larger sample of extracellular recordings of single-unit responses to the same sounds. We could not measure directly the input SNR in these responses, so we instead examined the relationship between output correlations and output SNR. Because output SNR was a monotonic function of input SNR in all of our intracellular recordings, we expected that the same dependencies that we observed between output correlations and input SNR would also be evident when comparing output correlations and output SNR. Indeed, the effects of SNR on correlations observed extracellularly mirrored those observed intracellularly: as shown in Figure $2 E, F$, the best linear fit between output SNR and output signal correlation had a positive slope for all pairs $(n=34)$, while the best linear fit between output SNR and output noise correlation had a negative slope for all but two pairs.

We next examined the relationship between input signal correlation and output noise correlation that resulted from changing the correlation between the signal components of the sounds while keeping the SNR and the correlation in the noise components of the sounds fixed. The output noise correlation as a function of input signal correlation for a typical intracellular recording is shown in Figure $2 G$; as expected from the results of our in vitro experiments, output noise correlation increased with increasing input signal correlation. This effect was consistent across all of our intracellular recordings $(n=6$; Fig. $2 H)$ and was also evident in a larger sample of extracellular recordings of responses to the same sounds in which output noise correlation increased with output signal correlation for all pairs $(n=$ 34; Fig. $2 I, J)$.

\section{The impact of nonlinear correlation transfer of depends on cortical state}

We have demonstrated that nonlinear interactions between signal and noise inputs can significantly affect the transfer of signal and noise correlations in cortical neurons. However, the effects that we have demonstrated may depend on the regime in which the cortex is operating. In asleep, anesthetized, and awake animals, the state of the cortex can vary along a continuum of synchronized and desynchronized states across which the trialto-trial variability and correlations in sensory responses can vary dramatically (Steriade et al., 2001; Castro-Alamancos, 2004; Haider and McCormick, 2009; Harris and Thiele, 2011). When the cortex is in a synchronized state, concerted fluctuations between intrinsically generated up and down states result in sensory responses with low SNRs and large noise correlations, but in desynchronized states, up and down states are suppressed and sensory responses have higher SNRs and smaller noise correlations (Harris and Thiele, 2011; Marguet and Harris, 2011; Ecker et al., 2014).

The nature of variance gain and correlation gain suggests that these state-dependent differences in SNRs and noise correlations will affect the strength of the nonlinear interactions between signal and noise inputs. For example, because variance gain is sublinear, the effects of a change in input SNR on output correlations should be large when the SNR is low and small when the SNR is high. Similarly, because correlation gain is supralinear, the effects of a change in input signal correlation on output noise correlation should be small when noise correlations are small and large when noise correlations are large. Thus, we hypothesized that the effects of both variance gain and correlation gain should be strong when the cortex is in a synchronized state with low SNR and large noise correlations, and weak when the cortex is in a desynchronized state with high SNR and small noise correlations. To test whether the strength of the effects of nonlinear interactions between signal and noise inputs were indeed state dependent, we used a multitetrode array to record extracellular responses to speech (Fig. $3 A$ ) from populations of single units in A1 of gerbils anesthetized with either fentanyl, medetomidine, and midazolam (four populations, 147 neurons), which produce stable desynchronized cortical states, or ketamine and xylazine (five populations, 160 neurons), which produce stable synchronized cortical states (Pachitariu et al., 2014). Example responses from typical populations in synchronized and desynchronized A1 are shown in Figure 3B, and the distributions of SNRs and correlations in the responses for all neurons are shown in Figure $3 C-E$. The SNR of responses in the desynchronized state was much higher than that in the synchronized state (median values: 0.3 and 0.04 ), while the noise correlations in the desynchronized state were much smaller than those in the synchronized state (median values: 0.003 and 0.1 ). 
A

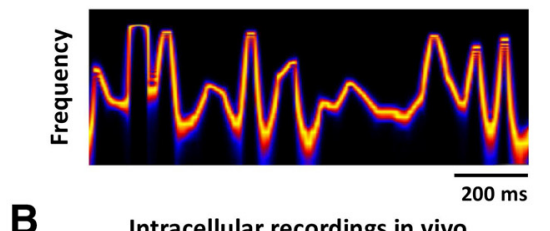

B Intracellular recordings in vivo
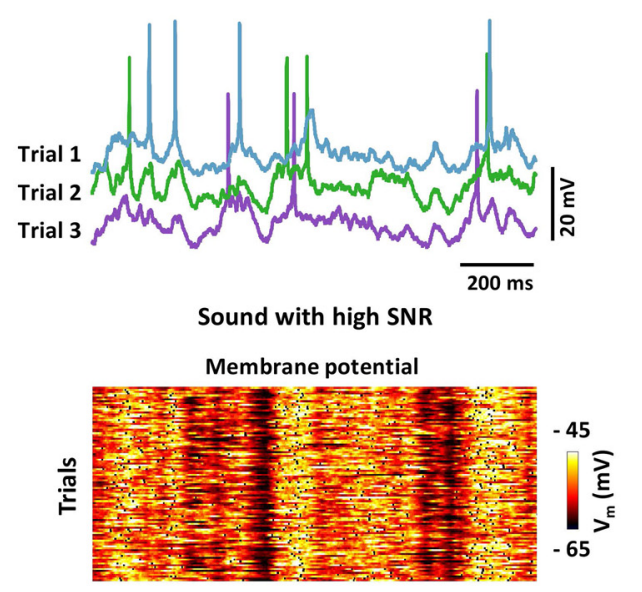

Spikes
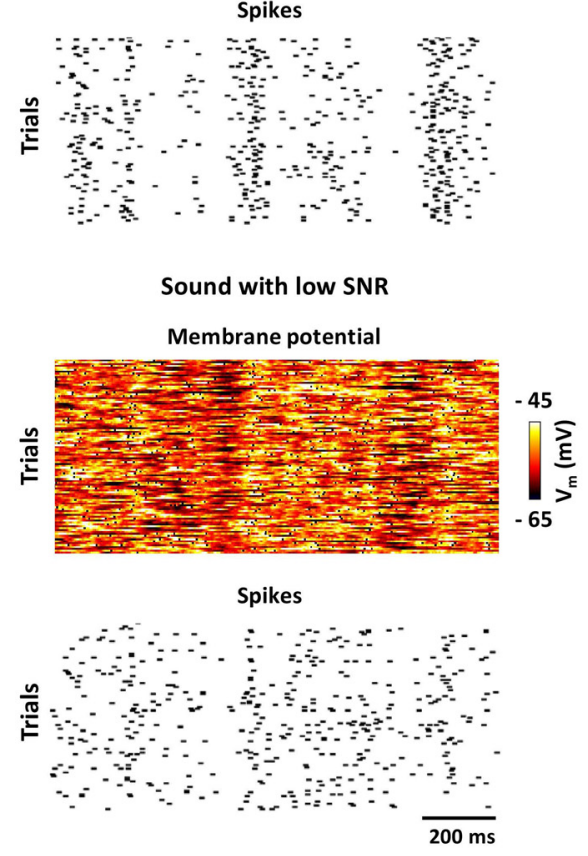

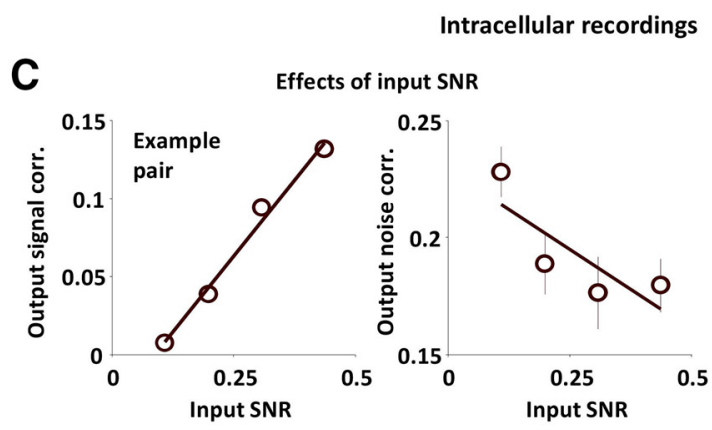

G Effects of input signal corr.
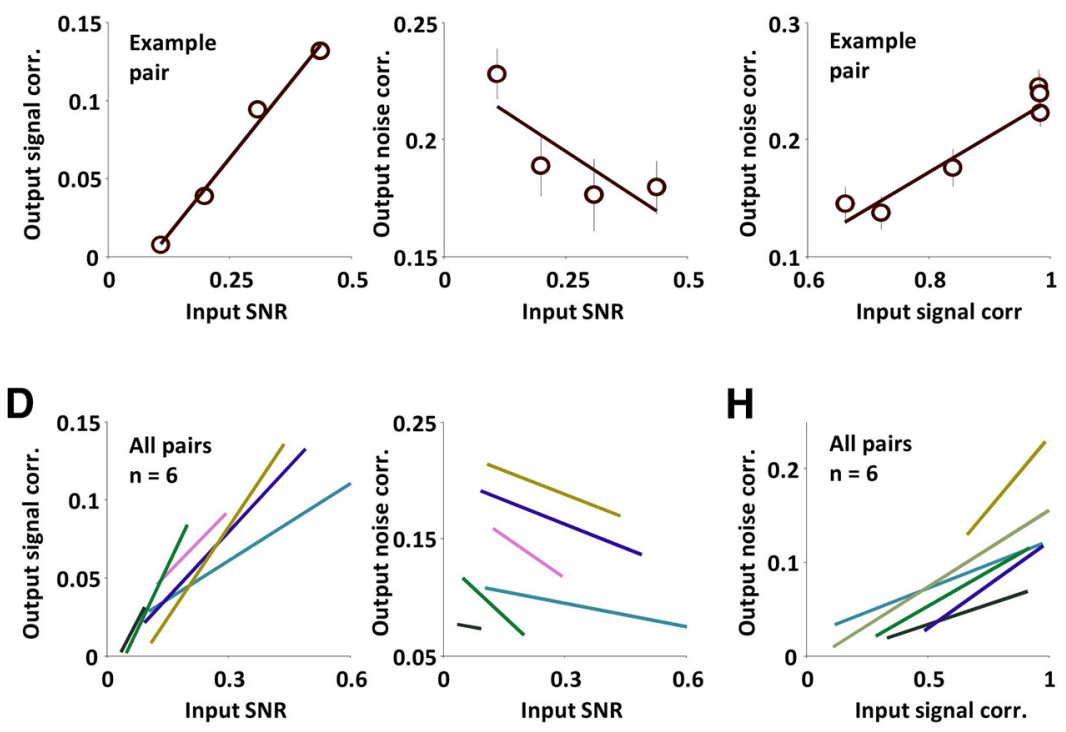

H

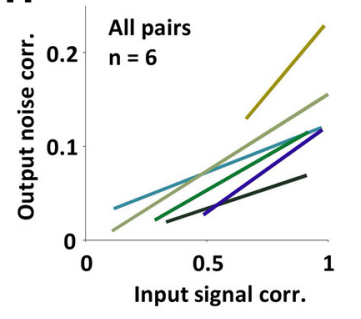

Extracellular recordings

$\mathbf{E}$

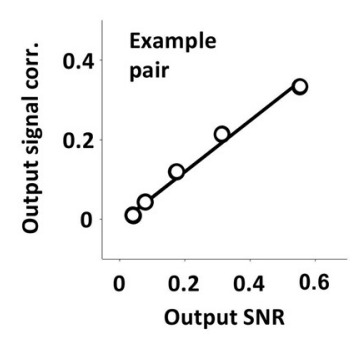

$\mathbf{F}$

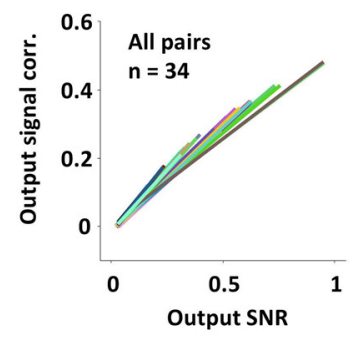

Effects of input SNR
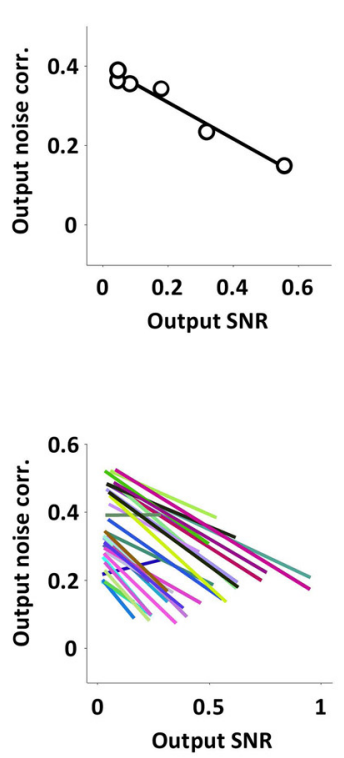

I Effects of input signal corr.

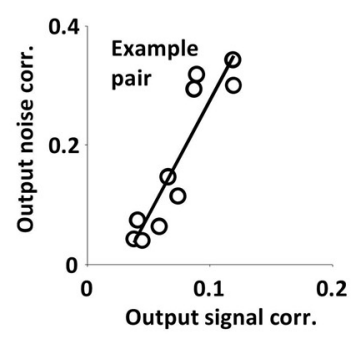

$\mathbf{J}$

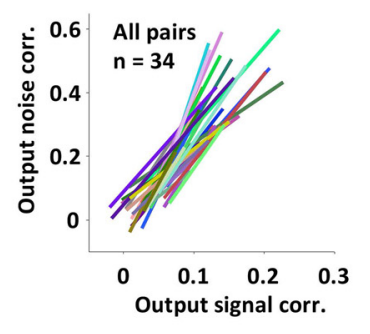

Figure 2. Nonlinear transfer of signal and noise correlations in vivo. $\boldsymbol{A}$, The spectrogram of sound with random FMs. See Materials and Methods for a detailed description of sound properties. $\boldsymbol{B}$, Example membrane potential and spiking responses of a typical neuron in gerbil A1 across trials for the same FM sound with two different sound SNRs. Action potentials were clipped for plotting. C, The output signal correlations (left) and output noise correlations (right) as a function of input SNR for the pair formed from the responses of a typical neuron to FM sounds with four different SNRs, along with the best linear fits. The input SNR for a pair of responses was computed as the geometric mean of the SNRs for each response. The error bars indicate the SDs of the distributions obtained from bootstrap resampling of trials. D. The best linear fits between input SNR and output signal correlation (left) and output noise correlation (right) for all intracellular recordings in our sample. $\boldsymbol{E}$, $\boldsymbol{F}$, Results for extracellular recordings plotted as in Cand $\boldsymbol{D}$, with input SNR replaced by output SNR. $\boldsymbol{G}$, The output noise correlation as a function of the input signal correlation for the pair formed from the responses of a typical neuron to FM sounds with different signal components, along with the best linear fit. $\boldsymbol{H}$, The best linear fits between input signal correlation and output noise correlation for all pairs in our sample. $\boldsymbol{I}, \boldsymbol{J}$, Results for extracellular recordings plotted as in $\mathbf{G}$ and $\boldsymbol{H}$, with input signal correlation replaced by output signal correlation.

Because responses in the synchronized state had lower SNRs and larger noise correlations than responses in the desynchronized state, the effects of nonlinear interactions between signal and noise inputs that we demonstrated above-the opposing dependencies of signal and noise correlations on SNR, and the positive dependency of noise correlation on signal correlationshould be more evident in the synchronized state than in the desynchronized state. Indeed, as shown in Figure 3F, scatter plots of the signal and noise correlations for all pairs, with dots colored by the geometric mean of the SNRs for each pair, suggest strong dependencies between SNR, signal correlation, and noise correlation in the synchronized state, while dependencies in the desynchronized state are much less evident. To quantify the strength of the dependencies in each state, we computed the partial correla- 
A Speech spectrogram

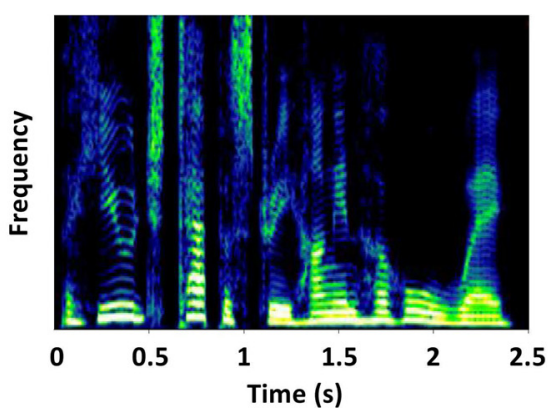

C

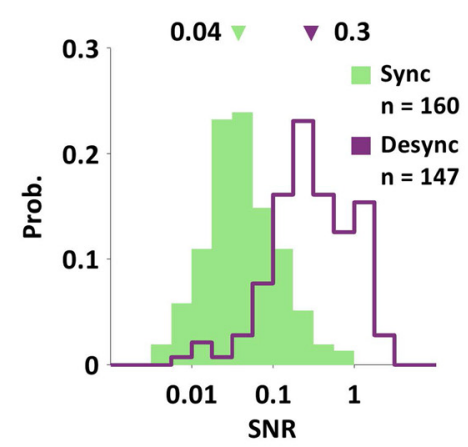

$\mathbf{F}$

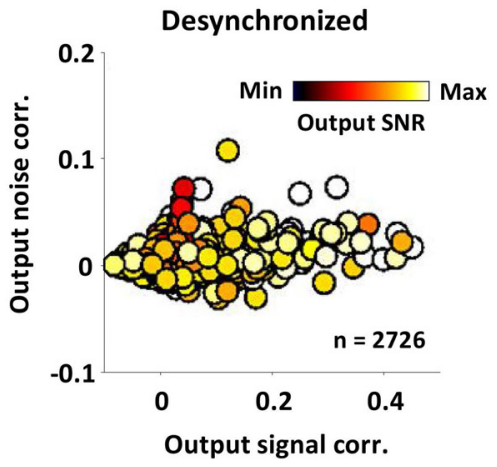

B

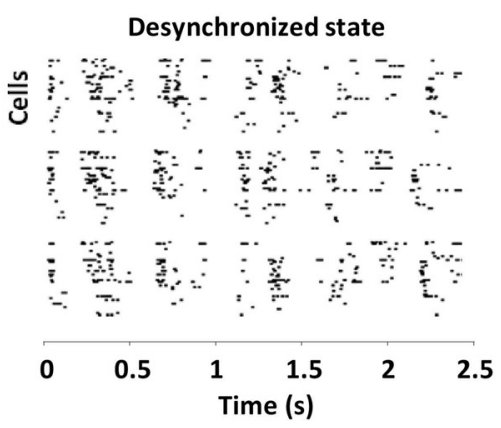

D

Output signal corr.

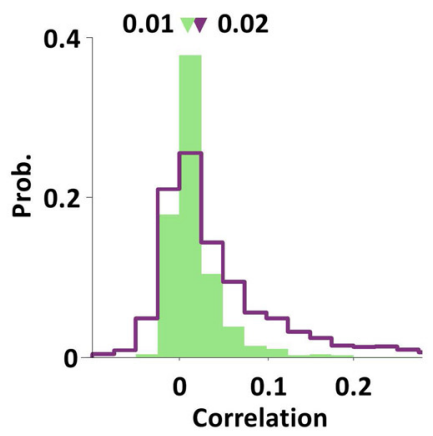

Synchronized

G

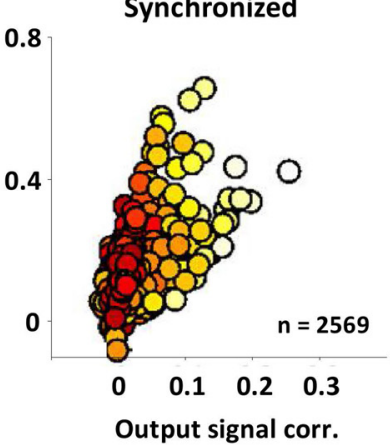

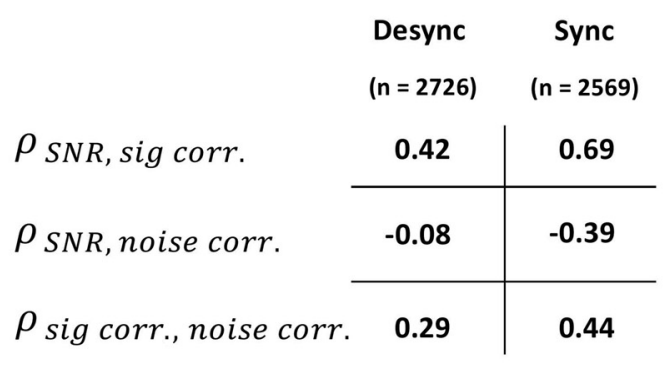

Figure 3. The impact of nonlinear correlation transfer depends on cortical state. $\boldsymbol{A}$, The spectrogram of a speech sound. $\boldsymbol{B}$, Raster plots showing the responses of typical A1 populations in desynchronized and synchronized states on three successive trials. Each row in the raster plot shows the spiking of one cell. $\boldsymbol{C}-\boldsymbol{E}$, The distributions of SNR, signal correlations, and noise correlations in the spiking of neurons in synchronized (green) and desynchronized (purple) A1. The median values are indicated by the arrows. All differences in population medians were significant with $p<$ 0.001 (Wilcoxon rank-sum test). $\boldsymbol{F}$, The spiking signal and noise correlations in the responses of simultaneously recorded pairs of neurons to speech in desynchronized and synchronized A1, with dots colored according to the geometric mean of the spiking SNR for each pair. G, Partial correlation coefficients between different spiking properties in responses of desynchronized and synchronized A1 to speech. The partial correlation between each possible pairing of SNR, signal correlation, and noise correlation was computed after factoring out the influence of the third quantity, as well as the influence of mean spike rate. All partial correlations were significant with $p<0.001$.

tion between each possible pairing of SNR, signal correlation, and noise correlation, while factoring out the influence of the third quantity, as well as the influence of mean spike rate, which can also affect correlations (de la Rocha et al., 2007). The results of this analysis, shown in Figure $3 G$, confirm that all three dependencies were significantly stronger in the synchronized state than in the desynchronized state (all partial correlations were significant with $p<0.001$ ).

Nonlinear transfer of signal and noise correlations in a simple model

We have attributed the effects of the interactions between signal and noise inputs on output correlations to thresholding, but the transformation of synaptic inputs to spiking output in real neurons also involves many other nonlinear processes. To confirm that thresholding alone was indeed sufficient to account for the effects that we observed, we examined correlation transfer in a DG model (Macke et al., 2009; Lyamzin et al., 2010, 2012). In the standard DG model (Fig. 4A), the response $r(t)$ is generated by thresholding a Gaussian process $v(t)$ with zero mean and variance $\sigma_{v}^{2}$ at each time step to create binary spike trains (Eq. 1):

$$
r(t)=\left\{\begin{array}{ccc}
1 & \text { if } & v(t)>1 \\
0 & & \text { else }
\end{array}\right.
$$

For a pair of neurons with the same input variance $\sigma_{v}^{2}$, the probability of any particular joint binary response is determined by $\sigma_{v}^{2}$ 
A

A dichotomized Gaussian (DG) model

$v(t)$

Input

Threshold

Output

(t) namply $\longrightarrow$ $\longrightarrow 0|1| 0|0| 0|1| r(t)$

C

$$
\text { Input Threshold Output }
$$

$\mathrm{s}(t)$

$n(t)$

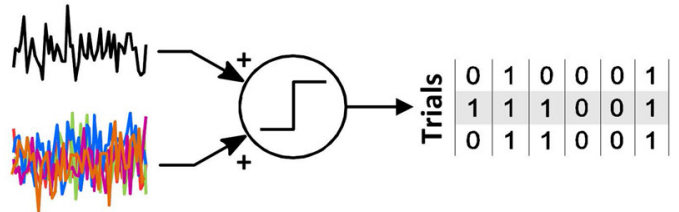

B

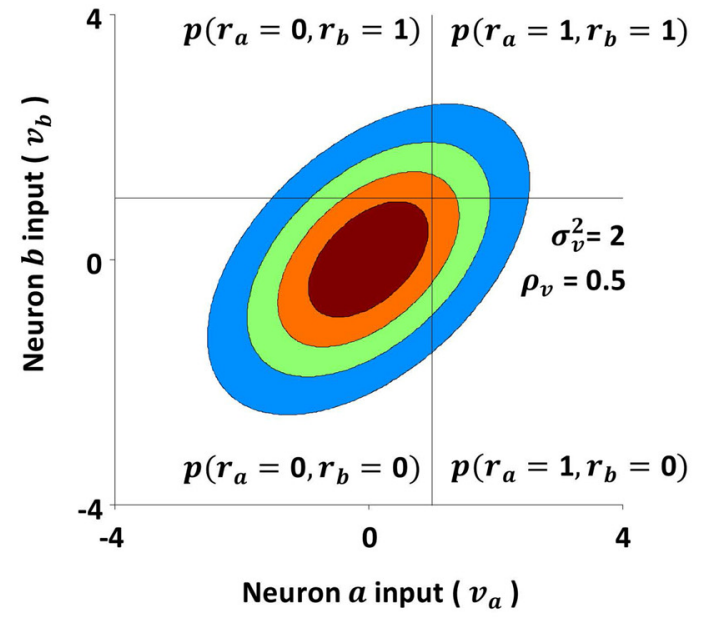

D

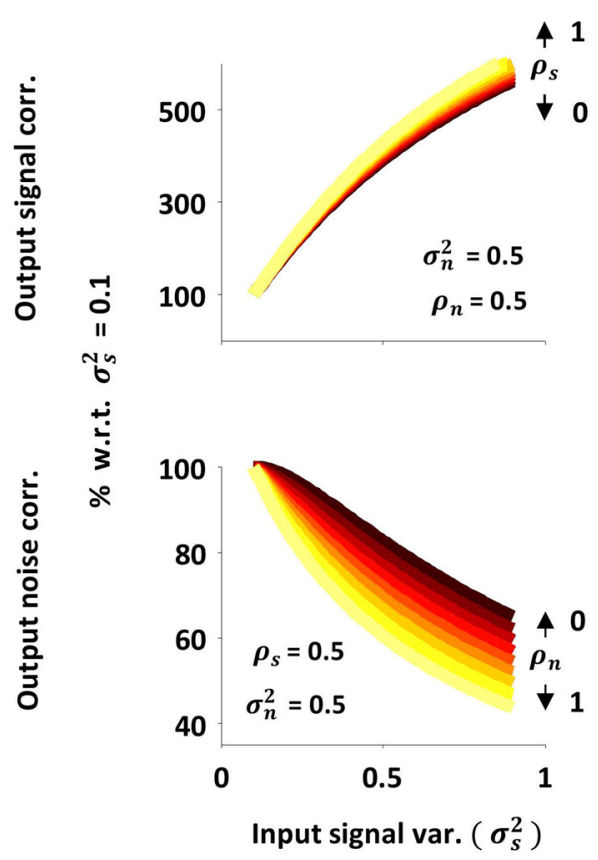

E Effects of input SNR

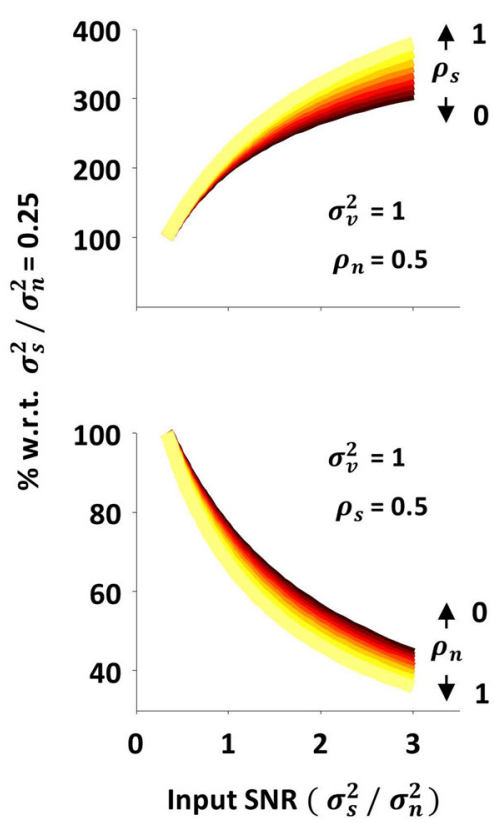

F Effects of input signal corr.

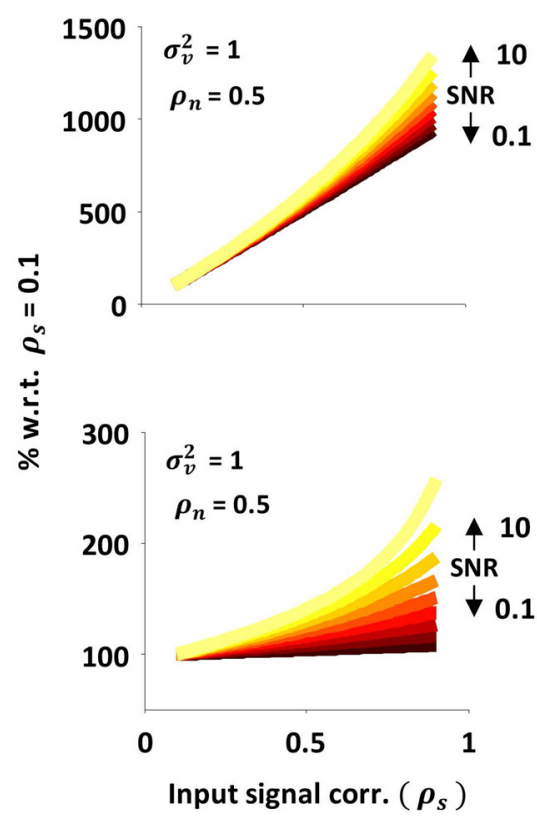

Figure 4. Nonlinear transfer of signal and noise correlations in a simple model. A-C, Schematic diagrams illustrating the DG model; see text for a detailed description. $\boldsymbol{D}$, The output signal correlations (top) and noise correlations (bottom) in DG model spiking as a function of input signal variance. Each colored line shows the output signal correlations for a different input signal correlation (top) or the output noise correlations for a different input noise correlation (bottom). Values are shown as percentage change relative to those for the smallest input signal variance tested. The values of the input properties that were fixed are shown on each panel. $\boldsymbol{E}, \boldsymbol{F}$, The output signal and noise correlations in DG model spiking as a function of input SNR and input signal correlation, plotted as in $\boldsymbol{D}$.

and the correlation between the inputs $\rho_{\mathrm{v}}$. This is illustrated in Figure $4 B$, which shows a bivariate Gaussian probability distribution with a positive correlation and the fractions of probability density corresponding to the four possible joint binary responses.

To capture signal and noise correlations, we extended the DG model (Fig. 4C) to have separate signal and noise inputs (Eq. 2):

$$
r(t)=\left\{\begin{array}{ccc}
1 & \text { if } & s(t)+n(t)>1 \\
0 & \text { else }
\end{array}\right.
$$

with signal input $s(t)$ with zero mean and variance $\sigma_{s}^{2}$ assumed to be the same on each trial, noise input $n(t)$ with zero mean and variance $\sigma_{n}^{2}$ assumed to be different on each trial, and correlations $\rho_{s}$ and $\rho_{n}$ between the signal and noise inputs.

We replicated our physiological experiments by examining the changes in the correlations of the extended DG model spiking that resulted from changes in different input properties. As shown in Figure $4 D-F$, spiking generated by the DG model showed the same interactions between signal and noise inputs that we observed in real neurons: changing input signal variance had opposing effects on output signal and noise correlations (Fig. $4 D$ ), as did changing input SNR (Fig. 4E), while increasing input signal correlation resulted in an increase in both output signal 


\section{Effects of input SNR}
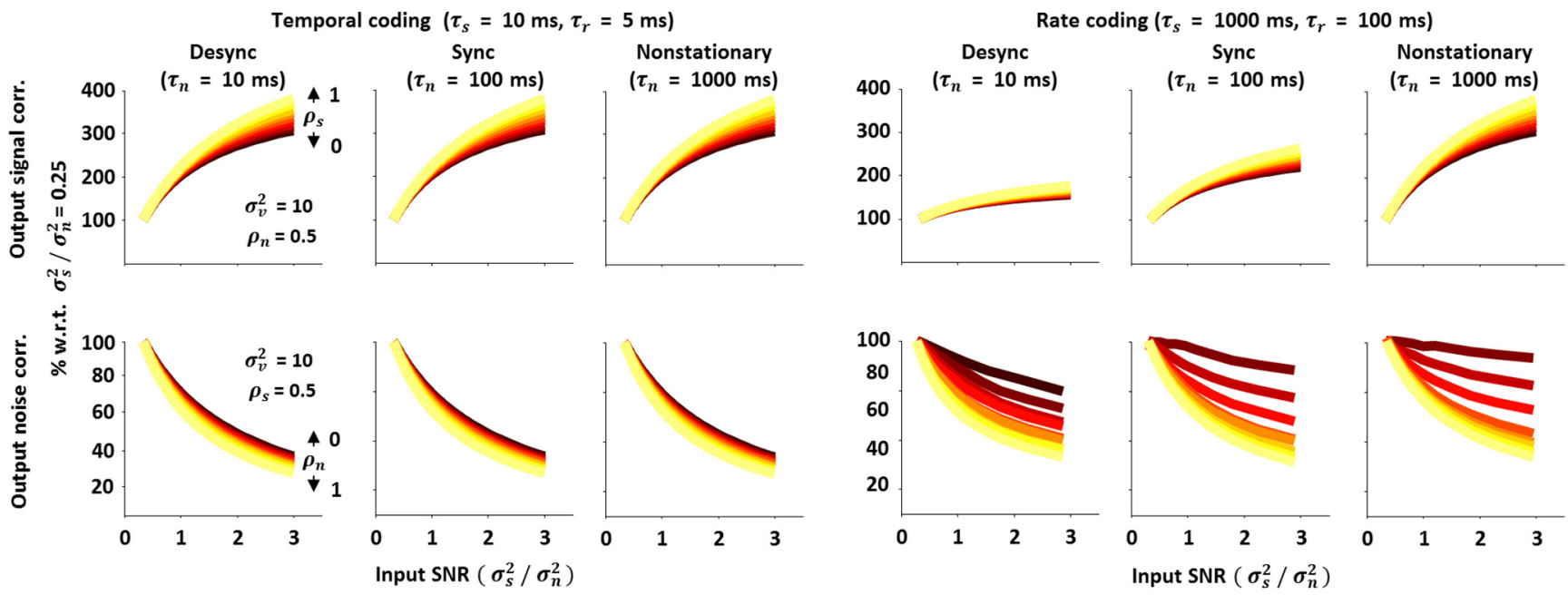

Effects of input signal corr.
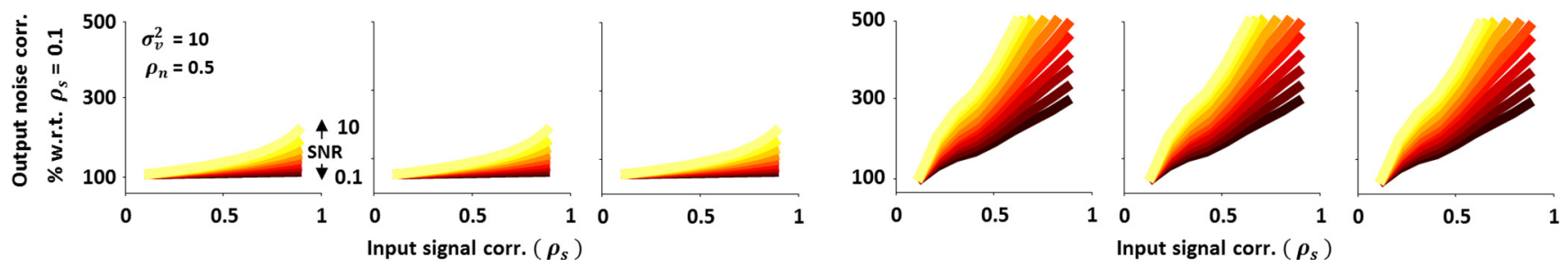

Figure 5. Nonlinear transfer of signal and noise correlations across different timescales. Top and middle rows, Output signal and noise correlations in the spiking of an LIF model as a function of input SNR for different input signal, noise, and response timescales, plotted as in Figure $4 E$. Bottom row, Output noise correlation in the spiking of an LIF model as a function of input signal correlation for different input signal, noise, and response timescales, plotted as in the bottom panel of Figure $4 F$.

and noise correlations (Fig. 4F). While these results do not explicitly rule out contributions from other nonlinear processes in real neurons, they do demonstrate that thresholding alone is sufficient to account for the interactions between signal and noise correlations that we observed.

\section{Nonlinear transfer of signal and noise correlations across different timescales}

Our experiments and analysis thus far have focused on correlations measured over relatively short timescales $(50 \mathrm{~ms}$ time bins for physiological experiments, or single time bins for the DG model). However, there are many regimes in which activity in cortical networks is correlated over much longer timescales. For example, in a temporal coding regime with precise spike timing, the relevant timescale for signal correlations may be short, but in a rate coding regime, the relevant timescale for signal correlations may be as long as several seconds. The relevant timescale for noise correlations can also vary over a wide range, depending on whether, for example, the cortex is in a synchronized state, with activity dominated by slow global fluctuations, or is in a desynchronized state, in which these fluctuations are suppressed.

To examine the nonlinear transfer of signal and noise correlations across a range of timescales, we used a LIF model, where the membrane potential $v(t)$ at each time step is given by the following (Eq. 3):

$$
\tau_{m} \frac{d v(t)}{d t}=-v(t)+s(t)+n(t)
$$

and generated binary spike trains as in the following DG model (Eq. 4):

$$
r(t)=\left\{\begin{array}{ccc}
1 & \text { if } & v(t)>1 \\
0 & & \text { else }
\end{array}\right.
$$

We used a membrane time constant $\tau_{\mathrm{m}}=10 \mathrm{~ms}$ and added temporal correlations to the signal and noise inputs so that input signal $s$ had autocorrelation $e^{-t / \tau_{s}}$ and cross-correlation $\rho_{s} e^{-t / \tau_{s}}$, and the input noise $n$ had autocorrelation $e^{-t / \tau_{n}}$ and crosscorrelation $\rho_{n} e^{-t / \tau_{n}}$. We found that the two main effects of nonlinear interactions between signal and noise inputs that we have focused on-the opposing changes in output signal and noise correlations resulting from a change in input SNR, and the increase in output noise correlation resulting from an increase in input signal correlation - were evident across all input signal and noise correlation timescales, as well as across all response timescales $\tau_{r}$ (the number of bins over which spike counts were summed before computing output correlations).

Figure 5 shows the changes in output correlations that resulted from changing input SNR or input signal correlation for different signal, noise, and response timescales. The top and middle rows show changes in output signal and noise correlations as a function of input SNR (Fig. 4E). The change in output signal correlation with increasing input SNR was always positive, while the change in output noise correlation was always negative. The strength of these effects was the same across all noise timescales in a temporal coding regime with fast signal and response timescales; however, in a rate coding regime with slow signal and 
response timescales, the strength of the changes in output signal correlation with changing input SNR increased with increasing noise timescale, while the strength of the changes in output noise correlation decreased (though this decrease was only evident for inputs with weak signal correlation). The bottom row of Figure 5 shows the changes in output noise correlation with increasing input signal correlation (Fig. $4 F$, bottom), which were always positive. The strength of the effects of input signal correlation on output noise correlation increased with increasing input signal and response timescale, but was independent of the input noise timescale. These results, while confirming that the effects of spike threshold on the transfer of signal and noise correlations are qualitatively similar across all input and output timescales, also demonstrate that the strength of the effects may be timescale dependent.

\section{The impact of nonlinear correlation transfer on the coding of sensory information}

We have shown that the nonlinear transfer of signal and noise correlations is a general phenomenon with similar effects in many different contexts, but we have not yet examined its impact on sensory coding. To measure the efficiency of coding, we calculated the mutual information between the input signal and output spiking of the extended DG model (Eq. 2), which quantifies the degree to which the observation of output spiking reduces uncertainty about the value of the input signal. We consider first the effects of a change in input SNR on output signal and noise correlations. Because a change in input SNR causes opposing changes in output signal and noise correlations, the overall impact of these changes on information is difficult to predict. Without nonlinear correlation transfer, changes in input SNR would have no effect on output correlations. Thus, to measure the impact of the dependence of output correlations on input SNR, we can vary the input SNR and compare the information in output spiking under normal conditions (i.e., when output correlations change with input SNR) with the information in output spiking when output correlations are artificially fixed. This comparison cannot be made with experimental data, as there is no way to decouple input SNR and output correlations in real neurons; however, it can be made using the DG model, as the SNR and correlations in the output spiking of the model can be manipulated independently (this is achieved not by removing the effects of nonlinear correlation transfer from the model, but rather by compensating for them; see Materials and Methods).

The red line in Figure $6 \mathrm{~A}$ shows the information in single time bins (equivalent to the information in the PSTH; Brenner et al., 2000) as a function of input SNR with fixed input signal and noise correlations. As expected, information increased strongly with increasing input SNR. However, without the effects of nonlinear correlation transfer, when output correlations were artificially fixed at their values for the lowest input SNR (black line), the increase in information with increasing input SNR was even stronger. Thus, the changes in output signal and noise correlations with increasing input SNR due to nonlinear correlation transfer reduce information.

To gain further insight into how the effects of input SNR on output correlations affect information, we can break the total information down into separate components related to signal and noise correlations (Pola et al., 2003). The contribution of signal correlation to the information is shown in Figure $6 B$ (note that this contribution is always negative, as signal correlation can only be a source of redundancy). At the lowest input SNR, the contribution of signal correlation to the information is small (because the output signal correlation is small). The negative impact of signal correlation increases when output signal correlation increases with increasing SNR (red), but not when output correlations are fixed (black).

The contribution of noise correlation to the total information is shown in Figure 6C. This contribution is negative when output correlations change with increasing input SNR (red), and positive when output correlations are fixed (black). Thus, the decrease in output noise correlation with increasing input SNR is accompanied by a decrease in information. This result may seem surprising; since noise correlations are often thought to be detrimental to coding, one might expect a decrease in noise correlation to result in an increase in information. However, the impact of noise correlations on coding is not necessarily straightforward, as noise correlations can have both stimulus-independent and stimulus-dependent components. The contribution of stimulusindependent noise correlations to information can be either positive or negative: if signal and noise correlations have opposite signs, this contribution will be positive, whereas if signal and noise correlations have the same sign, this contribution will be negative (Panzeri et al., 1999). Since we consider only positive signal and noise correlations, the stimulus-independent contribution of noise correlations to information is always negative. As shown in Figure 6D, the negative impact of stimulus-independent noise correlation increases when output correlations change with increasing input SNR (red), but not when output correlations are fixed (black).

The stimulus-dependent contribution of noise correlation to the total information is shown in Figure 6E. This contribution can only be positive (differences in the noise correlations associated with different stimuli help to distinguish the responses to those stimuli). The contribution of stimulus-dependent noise correlation to information remains constant as output correlations change with increasing input SNR (red), but increases with increasing input SNR when output correlations are fixed (black). The positive impact of stimulus-dependent noise correlations when input SNR increases and output correlations are fixed is due to the fact that, although the average output noise correlation over all stimuli does not change, the range of output noise correlations for individual stimuli, and, consequently, the stimulus dependence of noise correlations, increases as the input signal variance is increased; when output noise correlations are not fixed and decrease with increasing SNR, this effect is offset. The results in Figure $6 A-E$ show that information is decreased by the changes in output correlations with increasing input SNR through a combination of effects related to changes in signal correlation, stimulusindependent noise correlation, and stimulus-dependent noise correlation.

The same approach can be used to measure the impact of the effects of input signal correlation on output noise correlation. Without nonlinear interactions between signal and noise inputs, changes in input signal correlation would have no effect on output noise correlation. Thus, to measure the impact of the dependence of output noise correlation on input signal correlation, we can vary the input signal correlation and compare the change in information in output spiking under normal conditions (i.e., when output noise correlation changes with input signal correlation) with the change in information in output spiking when output noise correlation is artificially fixed.

The red line in Figure $6 F$ shows the information in DG model spiking as a function of input signal correlation with fixed input $\mathrm{SNR}$ and input noise correlation. Information decreased strongly 
Effects of input SNR
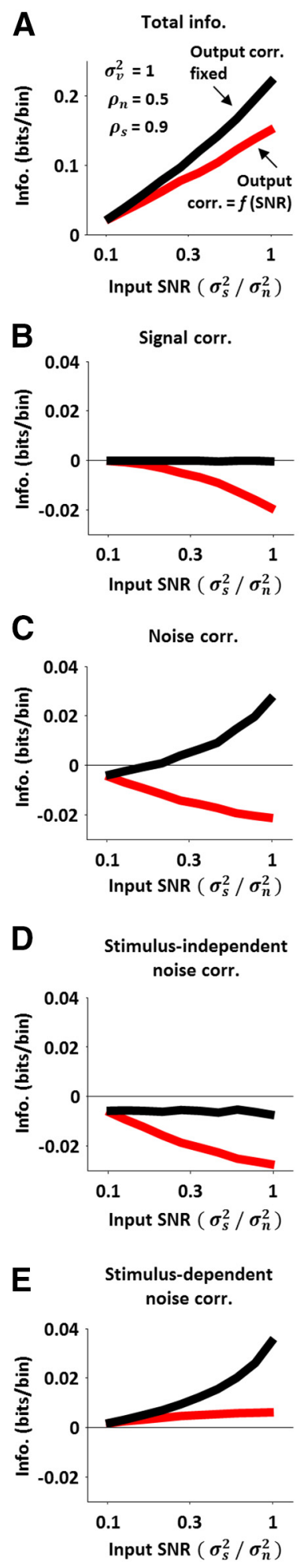

Effects of input signal corr.
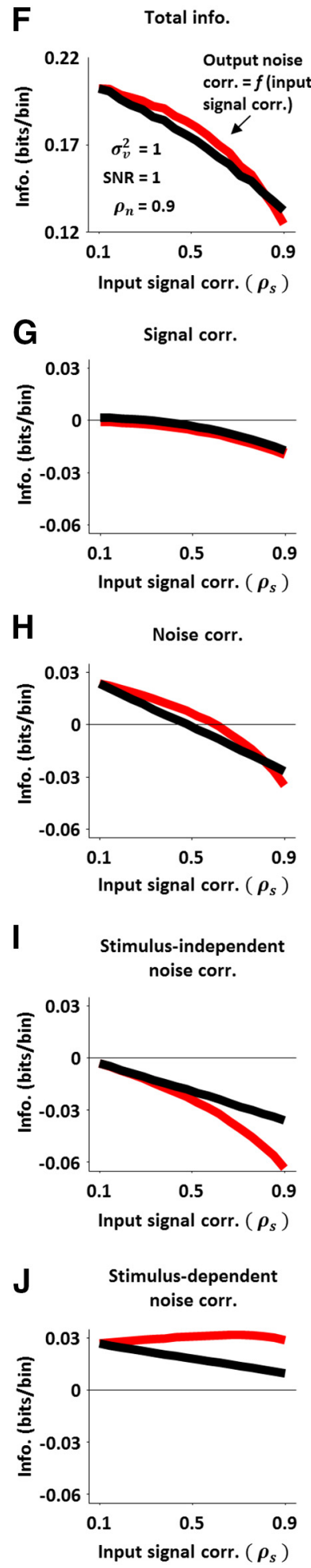

Figure 6. The impact of nonlinear correlation transfer on the coding of sensory information. $\boldsymbol{A}$, The information in DG model spiking as a function of input SNR with (red) and without (black) the effects of input SNR on output correlations. The red line was obtained by measuring the information in DG model spiking for different input SNRs with the input signal and noise correlations fixed at the values indicated on the panel. The black line was obtained by measuring the information in DG model spiking for different input SNRs with the input signal and noise correlations varied to compensate for changes in input SNR such that the output correlations remained fixed at their values for the lowest input SNR. $\boldsymbol{B}-\boldsymbol{E}$, The contribution of signal correlation, noise correlation, stimulus-independent noise correlation, and stimulus-dependent noise correlation to the information in DG model spiking with (red) and with increasing signal correlation, as expected, but the effects of removing nonlinear interactions between signal and noise inputs by artificially fixing the output noise correlation at its value for the lowest input signal correlation (black line) were relatively small. The change in output noise correlation with increasing input signal correlation was relatively large (from 0.25 for lowest input signal correlation to 0.45 for the highest input signal correlation), so it may seem surprising that fixing the output noise correlation resulted in only a small change in information; however, breaking down the information into separate components related to signal and noise correlations revealed relatively large, but offsetting, changes in different components.

Because the negative impact of signal correlation is independent of the output noise correlation (Fig. 6G), the differences in information with and without the effects of input signal correlation on output noise correlation are due exclusively to the contribution of noise correlation (Fig. $6 H$ ). The negative impact of stimulus-independent noise correlation (Fig. 6I) increases more rapidly when output noise correlation increases with increasing input signal correlation (red) than when output noise correlation is fixed (black), while the positive impact of stimulus-dependent noise correlation (Fig. $6 \mathrm{~J}$ ) increases slightly as output noise correlation increases with increasing input signal correlation (red), but decreases with increasing input signal correlation when output noise correlation is fixed (black). Thus, the effects of input signal correlation on output noise correlation have a relatively small impact on the total information because the changes in the contributions of stimulus-dependent and stimulus-independent noise correlations due to these effects have opposing signs and, thus, offset each other. Together, the results in Figure 6 demonstrate that spike threshold plays a major role in shaping both stimulusindependent and stimulus-dependent noise correlations, and that the interactions between these different forms of noise correlation can have important consequences for sensory coding.

\section{The origin of nonlinear correlation transfer}

Finally, we can use the DG model to gain insight into how the changes in correlations from membrane potential to spiking arise. Figure $7 A$ shows the nonlinear relationship between input and output correlation that is characteristic of correlation gain; assuming positive correlations, the output correlation increases supralinearly as the input correlation is increased. We can gain insight into the origin of correlation gain by analyzing the factors that determine the transfer of correlation from input to output within the standard DG model (Eq. 1). If we consider two neurons $a$ and $b$ with the same input variance $\sigma_{v}^{2}$ and positive input correlation $\rho_{v}$, then the output correlation $\rho_{r}$ can be written in terms of Gaussian CDFs (Eq. 5):

\section{$\leftarrow$}

without (black) the effects of input SNR on output correlations, plotted as function of input SNR. $\boldsymbol{F}$, The information in DG model spiking as a function of input signal correlation with (red) and without (black) the effects of input signal correlation on output noise correlation. The red line was obtained by measuring the information in DG model spiking for different input signal correlations with the input SNR and noise correlation fixed at the values indicated on the panel. The black line was obtained by measuring the information in DG model spiking for different input signal correlations with the input noise correlation varied to compensate for changes in input signal correlation such that the output noise correlation remained fixed at its value for the lowest input signal correlation. G-J, The contribution of signal correlation, noise correlation, stimulus-independent noise correlation, and stimulus-dependent noise correlation to the information in DG model spiking with (red) and without (black) the effects of input signal correlation on output noise correlation, plotted as function of input signal correlation. 


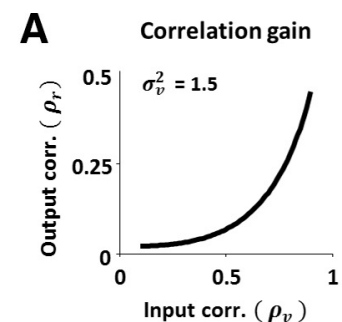

C

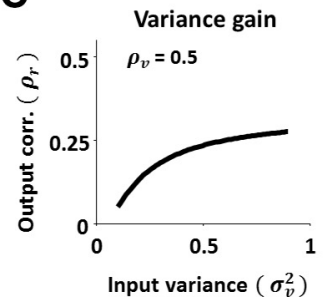

D

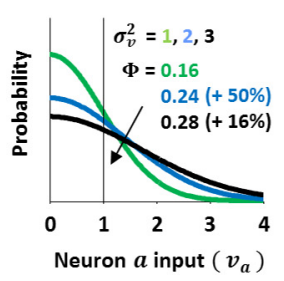

B

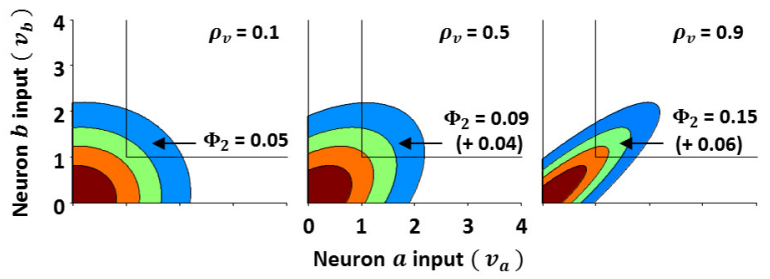

E

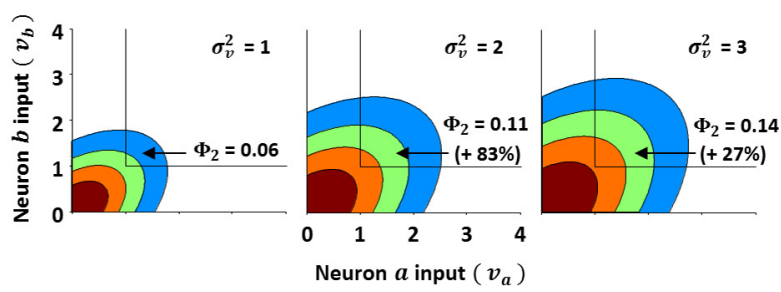

Figure 7. The origin of nonlinear correlation transfer. $\boldsymbol{A}, \mathrm{A}$ schematic diagram of correlation gain. $\boldsymbol{B}$, Schematic diagrams of the change in a bivariate Gaussian probability distribution with changing correlation. The probability that a sample from the distribution takes on a value $>1$ on both dimensions $\left(\Phi_{2}\right)$ is indicated on each panel, along with the change in this probability relative to that corresponding to the next lowest correlation. $C$, A schematic diagram of variance gain. $D, A$ schematic diagram of the change in a univariate Gaussian probability distribution with changing variance. The probability that a sample from the distribution takes on a value $>1(\Phi)$ is indicated on each panel, along with the change in this probability relative to that corresponding to the next lowest variance. $\boldsymbol{E}$, Schematic diagrams of the change in a bivariate Gaussian probability distribution with changing variance. The probability that a sample from the distribution takes on a value $>1$ on both dimensions $\left(\Phi_{2}\right)$ is indicated on each panel, along with the change in this probability relative to that corresponding to the next lowest variance.

$$
\begin{aligned}
& \rho_{r}=\frac{\operatorname{cov}\left(r_{a}, r_{b}\right)}{\sqrt{\operatorname{var}\left(r_{a}\right) \operatorname{var}\left(r_{b}\right)}}=\frac{\operatorname{cov}\left(r_{a}, r_{b}\right)}{\operatorname{var}\left(r_{a}\right)}=\frac{\mathrm{E}\left[r_{a} r_{b}\right]-\mathrm{E}\left[r_{a}\right] \mathrm{E}\left[r_{b}\right]}{\mathrm{E}\left[r_{a}^{2}\right]-\mathrm{E}\left[r_{a}\right]^{2}} \\
& =\frac{\Phi_{2}\left(-1, \Sigma_{v}\right)-\Phi\left(-1, \sigma_{v}^{2}\right)^{2}}{\Phi\left(-1, \Sigma_{v}^{2}\right)-\Phi\left(-1, \sigma_{v}^{2}\right)^{2}}
\end{aligned}
$$

with input covariance matrix $\Sigma_{v}=\left[\begin{array}{cc}\sigma_{v}^{2} & \sigma_{v}^{2} \rho_{v} \\ \sigma_{v}^{2} \rho_{v} & \sigma_{v}^{2}\end{array}\right] . \Phi$ is the value of a univariate Gaussian CDF with zero mean and variance $\sigma_{v}^{2}$ evaluated at -1 , which is equivalent to the probability that a sample from the distribution has a value $>1$ (i.e., the probability of an individual neuron spiking in a single time bin). $\Phi_{2}$ is the value of a bivariate Gaussian CDF with zero mean and covariance $\Sigma_{v}$ evaluated at -1 , which is equivalent to the probability that a sample from the distribution has a value $>1$ on both dimensions (i.e., the probability of both neurons spiking together in a single time bin).

A change in the input correlation $\rho_{v}$ will change the $\Phi_{2}$ term in Eq. 5, but will have no effect on the $\Phi$ terms. Thus, the supralinear correlation gain must result from the fact that each increase in $\rho_{v}$ results in successively larger increases in $\Phi_{2}$ and, thus, successively larger increases in the output correlation $\rho_{r}$. Figure $7 B$, left, shows a bivariate Gaussian distribution with a weak correlation $\left(\rho_{v}=0.1\right)$. This distribution is nearly circular and only a relatively small fraction of probability density is above the threshold of 1 on both dimensions $\left(\Phi_{2}=0.05\right)$. When the correlation is increased to $\rho_{v}=0.5$, as shown in the middle panel, the distribution becomes slightly elongated and the fraction of probability density above threshold on both dimensions increases to $\Phi_{2}=0.09$ (a change of 0.04). When the correlation is increased again by the same amount to $\rho_{v}=0.9$, as shown in the right panel, the distribution becomes more elongated and the fraction of probability density above threshold on both dimensions again increases; however, this increase to $\Phi_{2}=0.15$ (a change of 0.06 ) is $50 \%$ larger than the previous increase in $\Phi_{2}$, even though the change in the correlation $\rho_{v}$ was the same. This effect, which underlies correlation gain, results from the fact that each successive elongation of the probability distribution results in successively more central parts of the distribution being above threshold.

We can use the same approach to gain insight into the origin of variance gain. The nonlinear relationship between input variance and output correlation that is characteristic of variance gain is shown in Figure $7 C$; the output correlation $\rho_{r}$ increases as the input variance $\sigma_{v}^{2}$ is increased, even when the input correlation $\rho_{v}$ is constant. Variance gain can be understood by comparing the $\Phi_{2}$ and $\Phi$ terms in Equation 5. Figure $7 D$ shows univariate Gaussian distributions with increasing variance $\left(\sigma_{v}^{2}=1,2,3\right)$ and the corresponding fractions of probability density above threshold ( $\Phi=0.16,0.24,0.28$, a change of $50 \%$ from $\sigma_{v}^{2}=1$ to $\sigma_{v}^{2}=2$, and a change of $16 \%$ from $\sigma_{v}^{2}=2$ to $\sigma_{v}^{2}=3$ ). Figure $7 E$ shows bivariate Gaussian distributions with the same three variances and the corresponding fractions of probability density above threshold $\left(\Phi_{2}=0.06,0.11,0.14\right.$, changes of 83 and $\left.27 \%\right)$. Because the impact of an increase in the variance $\sigma_{v}^{2}$ on the $\Phi_{2}$ term is relatively larger than the impact on the $\Phi$ terms, the output correlation $\rho_{r}$ increases. This effect, which underlies variance gain, arises from the elongation of the bivariate distribution caused by the positive correlation, which results in the same increase in variance shifting more probability density above threshold for the bivariate distribution than for the univariate distribution. Note the sublinearity of variance gain: each successive increase in input variance causes a smaller increase in output correlation.

The same approach can also be used to gain insight into the origin of the nonlinear interactions between signal and noise inputs that affect the transfer of signal and noise correlations in the extended DG model (Eq. 2). If we consider two neurons $a$ and $b$ with the same input signal and noise variances $\sigma_{s}^{2}$ and $\sigma_{n}^{2}$, and input signal and noise correlations $\rho_{s}$ and $\rho_{n}$, then the output total correlation $\rho_{r}^{\text {tot }}$ can be written in terms of Gaussian CDFs similar to those in Equation 5 (Eq. 6):

$$
\rho_{r}^{\text {tot }}=\frac{\operatorname{cov}\left(r_{a}, r_{b}\right)}{\sqrt{\operatorname{var}\left(r_{a}\right) \operatorname{var}\left(r_{b}\right)}}=\frac{\Phi_{2}\left(-1, \Sigma_{t o t}\right)-\Phi\left(-1, \sigma_{s}^{2}+\sigma_{n}^{2}\right)^{2}}{\operatorname{var}\left(r_{a}\right)}
$$


with input covariance matrix $\Sigma_{\text {tot }}=\left[\begin{array}{cc}\sigma_{s}^{2}+\sigma_{n}^{2} & \sigma_{s}^{2} \rho_{s}+\sigma_{n}^{2} \rho_{n} \\ \sigma_{s}^{2} \rho_{s}+\sigma_{n}^{2} \rho_{n} & \sigma_{s}^{2}+\sigma_{n}^{2}\end{array}\right]$. Since we consider only changes in signal and noise inputs that do not affect the total variance $\sigma_{v}^{2}$, we can ignore the variance term in the denominator and restrict our focus to the $\Phi$ and $\Phi_{2}$ terms in the numerator.

The output signal correlation, which we define as the correlation between spiking recorded on different trials, can be written as follows (Eq. 7):

$$
\rho_{r}^{s i g}=\frac{\operatorname{cov}^{*}\left(r_{a}, r_{b}\right)}{\sqrt{\operatorname{var}\left(r_{a}\right) \operatorname{var}\left(r_{b}\right)}}=\frac{\Phi_{2}\left(-1, \Sigma_{s i g}\right)-\Phi\left(-1, \sigma_{s}^{2}+\sigma_{n}^{2}\right)^{2}}{\operatorname{var}\left(r_{a}\right)}
$$

where $\operatorname{cov}^{*}$ denotes the covariance between spiking recorded on different trials and $\Sigma_{s i g}=\left[\begin{array}{cc}\sigma_{s}^{2}+\sigma_{n}^{2} & \sigma_{s}^{2} \rho_{s} \\ \sigma_{s}^{2} \rho_{s} & \sigma_{s}^{2}+\sigma_{n}^{2}\end{array}\right]$. The only difference between Equations 6 and 7 is in the off-diagonal elements of the covariance matrices in the $\Phi_{2}$ terms; because noise inputs on different trials are uncorrelated (i.e., $\rho_{n}=0$ ), the $\sigma_{n}^{2} \rho_{n}$ term in $\Sigma_{\text {tot }}$ is absent from $\Sigma_{\text {sig. }}$. The output noise correlation, which we define as the difference between the total correlation and the signal correlation, can then be written as follows (Eq. 8):

$$
\rho_{r}^{\text {noise }}=\rho_{r}^{\text {tot }}-\rho_{r}^{\text {sig }}=\frac{\Phi_{2}\left(-1, \Sigma_{\text {tot }}\right)-\Phi_{2}\left(-1, \Sigma_{\text {sig }}\right)}{\operatorname{var}\left(r_{a}\right)}
$$

Using Equations 7 and 8, we can examine the effects of changes in the signal and noise inputs on output signal and noise correlation. The two main effects of nonlinear interactions between signal and noise inputs that we have focused on have been the opposing changes in output signal and noise correlations resulting from a change in input SNR, and the increase in output noise correlation resulting from an increase in input signal correlation. The origin of the effects of a change in input signal correlation on output noise correlation can be seen in Equation 8. If the input signal correlation $\rho_{s}$ is increased, the off-diagonal elements of the $\Sigma_{\text {tot }}$ and $\Sigma_{\text {sig }}$ terms will increase by the same amount. However, assuming the input noise correlation $\rho_{n}$ is positive, the offdiagonal elements of $\Sigma_{\text {tot }}$ will be larger than those of $\Sigma_{\text {sig }}$ to start with, so the resulting increase in the first $\Phi_{2}$ term in Equation 8 will be larger than that in the second $\Phi_{2}$ term because of correlation gain (i.e., for the same reasons described in the discussion of Fig. $7 B$ above). Thus, because an increase in input signal correlation increases the first $\Phi_{2}$ term more than the second $\Phi_{2}$ term, the output noise correlation, which is proportional to the difference between them, will increase.

The origin of the effects of a change in input SNR on output signal correlation can be seen in Equation 7. Assuming positive input signal and noise correlations $\rho_{s}$ and $\rho_{n}$, if the input SNR is increased by increasing $\sigma_{s}^{2}$ and decreasing $\sigma_{n}^{2}$ such that the total input variance $\sigma_{s}^{2}+\sigma_{n}^{2}$ does not change, the off-diagonal elements of $\Sigma_{\text {sig }}$ in Equation 7 will increase while the diagonal elements will remain the same. Consequently, the $\Phi_{2}$ term in Equation 7 will increase while the $\Phi$ term stays the same and, thus, the output signal correlation $\rho_{r}^{\text {sig }}$ will increase.

The origin of the effects of a change in input SNR on output noise correlation can be seen in Equation 8. In addition to changing the off-diagonal elements of $\Sigma_{\text {sig, }}$, the increase in input SNR will also change the off-diagonal elements of $\Sigma_{\text {tot }}$. However, because the increase in input SNR is achieved in part through a decrease in the noise variance $\sigma_{n}^{2}$, the change in the off-diagonal elements of $\Sigma_{\text {tot }}$, which include $\sigma_{n}^{2}$, can never exceed the change in the off-diagonal elements $\Sigma_{\text {sig, }}$, which do not. Thus, the change in the second $\Phi_{2}$ term of Equation 8 that results from an increase in input SNR will be greater than the corresponding change in the first $\Phi_{2}$ term, and the output noise correlation will decrease.

\section{Discussion}

Taken together, our in vitro, in vivo, and modeling results suggest that the threshold nonlinearity associated with spike generation can significantly transform the signal and noise correlations in cortical networks during sensory processing. Previous studies have shown that changes in input strength can affect output correlation, imposing a dependency between mean spike rate and total spiking correlation (de la Rocha et al., 2007). We have shown that even when total input strength is constant, the correlations in spiking can vary dramatically with changes in other input properties, with changes in the signal component of inputs affecting the noise component of outputs and vice versa. We found that changes in input SNR resulted in opposing changes in output signal and noise correlations, while increasing input signal correlations increased both output signal and noise correlations. These effects are due to nonlinear interactions between signal and noise inputs that could not be predicted by considering the effects of thresholding each input separately. Our modeling results demonstrate that a simple threshold nonlinearity is sufficient to reproduce the effects that we observed in vitro and in vivo, and that the effects of spike thresholding on signal and noise correlations are qualitatively (and, often, quantitatively) similar across a wide range of input and output timescales.

Our analysis of the information in DG model spiking suggests that the nonlinear transfer of signal and noise correlations has a significant impact on sensory coding, and that this impact is mediated through effects on both stimulus-independent and stimulus-dependent noise correlations. We found that the decrease in output noise correlation that accompanies an increase in input SNR reduces information, while the change in output noise correlation that accompanies a change in input signal correlation has little impact. These results may seem counterintuitive, as it is often reported that if both signal and noise correlations are positive (as in our study), noise correlation decreases information (Oram et al., 1998; Panzeri et al., 1999; Averbeck and Lee, 2006). This decrease is evident when considering the impact of noise correlation on the benefit achieved from averaging across responses to an input signal; in the extreme case in which all responses have identical noise, averaging has no effect at all. However, it is only stimulus-independent noise correlation that has the potential to be detrimental. In contrast, stimulusdependent noise correlation (i.e., noise correlation that changes when the input signal is changed) can only be beneficial; any change in the responses to different input signals can be used to distinguish those signals and, thus, provides information (Pola et al., 2003).

To separate the contributions of stimulus-independent and stimulus-dependent noise correlations to our results, we used an information breakdown approach (Pola et al., 2003). We found that the changes in output correlations that accompanied an increase in input SNR reduced information because of changes in both stimulus-independent and stimulus-dependent noise correlations, while the changes in output noise correlation that accompanied a change in input signal correlation had little impact on information because the changes in the contributions of stimulus-dependent and stimulus-independent noise correlations offset each other. These results demonstrate that the threshold nonlinearity associated with spike generation can serve as a source of strong stimulus-dependent noise correlation. 
Several theoretical studies have considered the impact of stimulus-dependent noise correlation on sensory coding in general (de la Rocha et al., 2007; Shea-Brown et al., 2008), but experimental studies have been limited to the visual and motor systems (Maynard et al., 1999; Montani et al., 2007; Ponce-Alvarez et al., 2013). Given that spike thresholding is a ubiquitous property of sensory systems, our results suggest that the impact of stimulusdependent noise correlation may be widespread and that further investigation in other systems and contexts is warranted. While our analysis was focused on neuronal pairs, investigation of the consequences of nonlinear correlation transfer for coding in larger populations where signal and noise correlations are predominantly positive, as in auditory cortex (Luczak et al., 2009; Pachitariu et al., 2015), is likely to reveal effects that are consistent with those we observed. However, the consequences of nonlinear correlation transfer in populations in which many pairs have signal and noise correlations that are negative, such as in motor cortex (Averbeck and Lee, 2006) and visual cortex (Ecker et al., 2014), may be more difficult to predict and require further study.

Many of the covariations in spiking properties that we observed have also been observed in other studies. Most studies of signal and noise correlations across cortical areas report a positive dependency, i.e., noise correlation tends to increase with increasing signal correlation (Bair et al., 2001; Averbeck and Lee, 2003; Kohn and Smith, 2005; Ecker et al., 2014) and, while it is generally assumed that this dependency reflects a corresponding dependency in input signal and noise correlations, our results demonstrate that this is not necessarily the case; we observed that an increase in input signal correlation caused an increase in both output signal and noise correlations, even when input noise correlation was constant. We also observed dependencies between SNR and correlations that are consistent with phenomena that have been reported in previous studies: for example, concurrent decreases in spiking trial-to-trial variability and noise correlations have been observed with changes in attention in V4 (Cohen and Maunsell, 2009; Mitchell et al., 2009) and with adaptation and changes in contrast and in V1 (Kohn and Smith, 2005). Our results indicate that nonlinear interactions between signal and noise inputs due to spike threshold may play a significant role in creating these dependencies.

Our results also show that, because the effects of variance gain decrease with increasing SNR and the effects of correlation gain increase with increasing noise correlation, the impact of spike threshold on signal and noise correlations depends strongly on brain state. We found that both the opposing dependencies of signal and noise correlations on SNR, and the positive dependency of noise correlation on signal correlation, were stronger in the synchronized state than in the desynchronized state. While both synchronized and desynchronized states are apparent in awake animals, the cortex often transitions to a desynchronized state during active processing (Crochet and Petersen, 2006; Greenberg et al., 2008; Poulet and Petersen, 2008; Xu et al., 2012; Luczak et al., 2013; Polack et al., 2013; Sachidhanandam et al., 2013; Tan et al., 2014; Zhou et al., 2014). In addition to increasing SNR and decreasing noise correlations, this transition appears to also decrease the nonlinearity of correlation transfer, making the correlation structure in cortical networks more robust to changes in input strength and, therefore, better able to faithfully encode correlations in sensory stimuli. Changes in brain state are not the only source of variation in the mean spike rates, trial-to-trial variability, and signal and noise correlations in cortical networks; the properties of sensory responses can also vary across a wide range due to changes in stimulus properties (Kohn and Smith, 2005; Hofer et al., 2011), adaptation (Gutnisky and Dragoi, 2008), or attention (Cohen and Maunsell, 2009; Mitchell et al., 2009). Thus, it may be critical to evaluate directly the role of spike threshold in shaping correlations in any given experimental context.

\section{References}

Averbeck BB, Lee D (2003) Neural noise and movement-related codes in the macaque supplementary motor area. J Neurosci 23:7630-7641. Medline

Averbeck BB, Lee D (2006) Effects of noise correlations on information encoding and decoding. J Neurophysiol 95:3633-3644. CrossRef Medline

Bair W, Zohary E, Newsome WT (2001) Correlated firing in macaque visual area MT: time scales and relationship to behavior. J Neurosci 21:16761697. Medline

Binder MD, Powers RK (2001) Relationship between simulated common synaptic input and discharge synchrony in cat spinal motoneurons. J Neurophysiol 86:2266-2275. Medline

Brenner N, Strong SP, Koberle R, Bialek W, de Ruyter van Steveninck RR (2000) Synergy in a neural code. Neural Comput 12:1531-1552. CrossRef Medline

Burak Y, Lewallen S, Sompolinsky H (2009) Stimulus-dependent correlations in threshold-crossing spiking neurons. Neural Comput 21:22692308. CrossRef Medline

Castro-Alamancos MA (2004) Dynamics of sensory thalamocortical synaptic networks during information processing states. Prog Neurobiol 74: 213-247. CrossRef Medline

Choi JH, Jung HK, Kim T (2006) A new action potential detector using the MTEO and its effects on spike sorting systems at low signal-to-noise ratios. IEEE Trans Biomed Eng 53:738-746. CrossRef Medline

Cohen MR, Kohn A (2011) Measuring and interpreting neuronal correlations. Nat Neurosci 14:811-819. CrossRef Medline

Cohen MR, Maunsell JH (2009) Attention improves performance primarily by reducing interneuronal correlations. Nat Neurosci 12:1594-1600. CrossRef Medline

Crochet S, Petersen CC (2006) Correlating whisker behavior with membrane potential in barrel cortex of awake mice. Nat Neurosci 9:608-610. CrossRef Medline

de la Rocha J, Doiron B, Shea-Brown E, Josić K, Reyes A (2007) Correlation between neural spike trains increases with firing rate. Nature 448:802806. CrossRef Medline

Dorn JD, Ringach DL (2003) Estimating membrane voltage correlations from extracellular spike trains. J Neurophysiol 89:2271-2278. Medline

Ecker AS, Berens P, Cotton RJ, Subramaniyan M, Denfield GH, Cadwell CR, Smirnakis SM, Bethge M, Tolias AS (2014) State dependence of noise correlations in macaque primary visual cortex. Neuron 82:235-248. CrossRef Medline

Gawne TJ, Richmond BJ (1993) How independent are the messages carried by adjacent inferior temporal cortical neurons? J Neurosci 13:2758-2771. Medline

Greenberg DS, Houweling AR, Kerr JN (2008) Population imaging of ongoing neuronal activity in the visual cortex of awake rats. Nat Neurosci 11:749-751. CrossRef Medline

Gu Y, Liu S, Fetsch CR, Yang Y, Fok S, Sunkara A, DeAngelis GC, Angelaki DE (2011) Perceptual learning reduces interneuronal correlations in macaque visual cortex. Neuron 71:750-761. CrossRef Medline

Gutnisky DA, Dragoi V (2008) Adaptive coding of visual information in neural populations. Nature 452:220-224. CrossRef Medline

Haider B, McCormick DA (2009) Rapid neocortical dynamics: cellular and network mechanisms. Neuron 62:171-189. CrossRef Medline

Happel MF, Jeschke M, Ohl FW (2010) Spectral integration in primary auditory cortex attributable to temporally precise convergence of thalamocortical and intracortical input. J Neurosci 30:11114-11127. CrossRef Medline

Harris KD, Thiele A (2011) Cortical state and attention. Nat Rev Neurosci 12:509-523. CrossRef Medline

Hazan L, Zugaro M, Buzsáki G (2006) Klusters, NeuroScope, NDManager: a free software suite for neurophysiological data processing and visualization. J Neurosci Methods 155:207-216. CrossRef Medline

Hofer SB, Ko H, Pichler B, Vogelstein J, Ros H, Zeng H, Lein E, Lesica NA, Mrsic-Flogel TD (2011) Differential connectivity and response dynamics of excitatory and inhibitory neurons in visual cortex. Nat Neurosci 14:1045-1052. CrossRef Medline

Jeanne JM, Sharpee TO, Gentner TQ (2013) Associative learning enhances 
population coding by inverting interneuronal correlation patterns. Neuron 78:352-363. CrossRef Medline

Kohn A, Smith MA (2005) Stimulus dependence of neuronal correlation in primary visual cortex of the macaque. J Neurosci 25:3661-3673. CrossRef Medline

Lampl I, Reichova I, Ferster D (1999) Synchronous membrane potential fluctuations in neurons of the cat visual cortex. Neuron 22:361-374. CrossRef Medline

Luczak A, Barthó P, Harris KD (2009) Spontaneous events outline the realm of possible sensory responses in neocortical populations. Neuron 62:413425. CrossRef Medline

Luczak A, Bartho P, Harris KD (2013) Gating of sensory input by spontaneous cortical activity. J Neurosci 33:1684-1695. CrossRef Medline

Lyamzin DR, Macke JH, Lesica NA (2010) Modeling population spike trains with specified time-varying spike rates, trial-to-trial variability, and pairwise signal and noise correlations. Front Comput Neurosci 4:144. CrossRef Medline

Lyamzin DR, Garcia-Lazaro JA, Lesica NA (2012) Analysis and modelling of variability and covariability of population spike trains across multiple time scales. Network 23:76-103. CrossRef Medline

Macke JH, Berens P, Ecker AS, Tolias AS, Bethge M (2009) Generating spike trains with specified correlation coefficients. Neural Comput 21:397-423. CrossRef Medline

Magri C, Whittingstall K, Singh V, Logothetis NK, Panzeri S (2009) A toolbox for the fast information analysis of multiple-site LFP, EEG and spike train recordings. BMC Neurosci 10:81. CrossRef Medline

Marguet SL, Harris KD (2011) State-dependent representation of amplitudemodulated noise stimuli in rat auditory cortex. J Neurosci 31:6414-6420. CrossRef Medline

Maynard EM, Hatsopoulos NG, Ojakangas CL, Acuna BD, Sanes JN, Normann RA, Donoghue JP (1999) Neuronal interactions improve cortical population coding of movement direction. J Neurosci 19:8083-8093. Medline

Middleton JW, Omar C, Doiron B, Simons DJ (2012) Neural correlation is stimulus modulated by feedforward inhibitory circuitry. J Neurosci 32: 506-518. CrossRef Medline

Mitchell JF, Sundberg KA, Reynolds JH (2009) Spatial attention decorrelates intrinsic activity fluctuations in macaque area V4. Neuron 63:879888. CrossRef Medline

Montani F, Kohn A, Smith MA, Schultz SR (2007) The role of correlations in direction and contrast coding in the primary visual cortex. J Neurosci 27:2338-2348. CrossRef Medline

Oram MW, Földiák P, Perrett DI, Sengpiel F (1998) The "Ideal Homunculus": decoding neural population signals. Trends Neurosci 21:259-265. CrossRef Medline

Pachitariu M, Lyamzin DR, Sahani M, Lesica NA (2015) State-dependent population coding in primary auditory cortex. J Neurosci 35:2058-2073. CrossRef Medline

Panzeri S, Schultz SR, Treves A, Rolls ET (1999) Correlations and the encoding of information in the nervous system. Proc Biol Sci 266:10011012. CrossRef Medline

Panzeri S, Senatore R, Montemurro MA, Petersen RS (2007) Correcting for the sampling bias problem in spike train information measures. J Neurophysiol 98:1064-1072. CrossRef Medline

Paxinos G, Franklin KB (2004) The mouse brain in stereotaxic coordinates: compact second edition. San Diego: Elsevier Academic.

Pola G, Thiele A, Hoffmann KP, Panzeri S (2003) An exact method to quantify the information transmitted by different mechanisms of correlational coding. Network 14:35-60. CrossRef Medline

Polack PO, Friedman J, Golshani P (2013) Cellular mechanisms of brain state-dependent gain modulation in visual cortex. Nat Neurosci 16:13311339. CrossRef Medline

Ponce-Alvarez A, Thiele A, Albright TD, Stoner GR, Deco G (2013) Stimulus-dependent variability and noise correlations in cortical MT neurons. Proc Natl Acad Sci U S A 110:13162-13167. CrossRef Medline

Poulet JF, Petersen CC (2008) Internal brain state regulates membrane potential synchrony in barrel cortex of behaving mice. Nature 454:881-885. CrossRef Medline

Sachidhanandam S, Sreenivasan V, Kyriakatos A, Kremer Y, Petersen CC (2013) Membrane potential correlates of sensory perception in mouse barrel cortex. Nat Neurosci 16:1671-1677. CrossRef Medline

Schmitzer-Torbert N, Jackson J, Henze D, Harris K, Redish AD (2005) Quantitative measures of cluster quality for use in extracellular recordings. Neuroscience 131:1-11. CrossRef Medline

Shamir M, Sompolinsky H (2004) Nonlinear population codes. Neural Comput 16:1105-1136. CrossRef Medline

Shea-Brown E, Josić K, de la Rocha J, Doiron B (2008) Correlation and synchrony transfer in integrate-and-fire neurons: basic properties and consequences for coding. Phys Rev Lett 100:108102. CrossRef Medline

Steriade M, Timofeev I, Grenier F (2001) Natural waking and sleep states: a view from inside neocortical neurons. J Neurophysiol 85:1969-1985. Medline

Tan AY, Chen Y, Scholl B, Seidemann E, Priebe NJ (2014) Sensory stimulation shifts visual cortex from synchronous to asynchronous states. Nature 509:226-229. CrossRef Medline

Tchumatchenko T, Malyshev A, Geisel T, Volgushev M, Wolf F (2010) Correlations and synchrony in threshold neuron models. Phys Rev Lett 104: 058102. CrossRef Medline

Xu S, Jiang W, Poo M-M, Dan Y (2012) Activity recall in a visual cortical ensemble. Nat Neurosci 15:449-455, S1-S2. CrossRef Medline

Zhou M, Liang F, Xiong XR, Li L, Li H, Xiao Z, Tao HW, Zhang LI (2014) Scaling down of balanced excitation and inhibition by active behavioral states in auditory cortex. Nat Neurosci 17:841-850. CrossRef Medline 